\title{
Research of Robust Trajectory Tracking Control and Attenuated Chattering: Application on Quadrotor
}

\author{
Guangli Zhou, Yongming Yao $(\mathbb{D}$, Huiying Liu, Xupeng Bai, and Jianbo Liu \\ School of Mechanical and Aerospace Engineering, Jilin University, Changchun 130022, China \\ Correspondence should be addressed to Yongming Yao; ymyao@jlu.edu.cn
}

Received 30 December 2020; Revised 19 April 2021; Accepted 29 May 2021; Published 8 June 2021

Academic Editor: Carlo Renno

Copyright $(2021$ Guangli Zhou et al. This is an open access article distributed under the Creative Commons Attribution License, which permits unrestricted use, distribution, and reproduction in any medium, provided the original work is properly cited.

In this paper, we presented a strategy for accurate trajectory tracking control of a quadrotor with unknown disturbances. To guarantee that the tracking errors of all system state variables converge to zero in finite time and eliminate the chattering phenomenon caused by the switching control action, a control strategy that combines linear prediction model of disturbances and fuzzy sliding mode control (SMC) based on logical framework with side conditions (LFSC) was designed. LFSC was applied for both position and attitude tracking of the quadrotor. Firstly, a linear prediction method was devised to minimize the effects of external disturbances. Secondly, a new fuzzy law was implemented to eliminate the chattering phenomenon. In addition, the stabilities of position and attitude were demonstrated by using Lyapunov theory, respectively. Simulation results and comprehensive comparisons demonstrated the superior performance and robustness of the proposed LFSC scheme in the case of external disturbances.

\section{Introduction}

The quadrotor, a typical unmanned aerial vehicle consisting of four symmetric propellers, has received much attention recently due to its low cost, easy maintenance, and potential for deployment in difficult environments [1]. The quadrotor design is the preferred choice for aerial robots, as quadrotor vehicles have vertical takeoff and landing capabilities and can hover at low speed [2]. As such, the quadrotor has been applied in many fields, including photography, education, transportation, and agriculture $[3,4]$. The quadrotor is a highly nonlinear, underactuated, and strongly coupled system; therefore, designing an effective control system for the quadrotor is a challenging task. However, the ability to minimize the effects of external disturbances is a bigger challenge [5].

Over the past several years, a number of advanced control strategies have been proposed to address the quadrotor trajectory tracking control problem [6]. Linear control strategies, including proportional integral derivative $[7,8]$, proportional derivative $[9,10]$, and linear quadratic [11] methods, have been applied successfully to improve stability; however, they are only effective over a small range around the operating point. If the quadrotor is subjected to greater external disturbances as it moves away from its control domain, system stability cannot be guaranteed [12]. Nonlinear control strategies can overcome the drawbacks of linear control methods to achieve good performance, even in harsh environments [13].

Sliding mode control (SMC) $[14,15]$ and backstepping control $[16,17]$ are the two among most widely used nonlinear control methods. Backstepping control is an efficient method for dealing with the trajectory tracking problem of the quadrotor via a nonlinear adaptive controller. However, backstepping control only provides sufficient stability when the disturbances are relatively constant or vary slowly over time. SMC utilizes a high-frequency switching control signal to enforce the system trajectories on the sliding surface, which has been studied for control of different underactuated systems [18-21]. The main properties of SMC are the proper transient performance and superior robust operation with the presence of model uncertainties and disturbances [22, 23]. Zhang et al. [24] adopted the adaptive recursive integral terminal sliding 
mode control to guarantee the convergence performance of the actual angle and the yaw rate with strong robustness and fast convergence rate. Zhou et al. [25] utilized deep learning method to compensate the uncertainties of the system without requirement of their upper bounds, which makes the designed switching gain much smaller. Song et al. [26] proposed a novel nonsingular fast-terminal sliding mode control method to facilitate the stabilization of nonlinear underactuated systems under disturbances. Gu et al. [27] utilized neural networks to approximate the lumped unknown dynamic model and designed a fast-terminal sliding mode control strategy to achieve the finite-time consensus tracking. Chen et al. [28] proposed a nonsingular terminal sliding mode control algorithm to implement accurate and robust body position trajectory tracking of six-legged robots. Xiong et al. [29] constructed a novel integral sliding mode surface to guarantee the synchronization error convergence to zero in finite time. However, the traditional SMC has the problem of chattering in the control signal which is undesirable [30]. Wang et al. [31] used adaptive integral SMC, backstepping, and terminal SMC to solve the trajectory problem; however, reducing chattering in the control input $\left(u_{2}, u_{3}, u_{4}\right)$ within the context of a hybrid finite-time control strategy is difficult to implement in practice. Mallavalli and Fekih [32] used SMC to solve the fault tolerance problem of the quadrotor; in this case, the gain of the switch function, sgn $(s)$, was a constant, resulting in substantial chattering. Wang et al. [33] proposed a terminal SMC, and Xinghuo Yu and Man Zhihong [34] proposed a fast-terminal SMC; however, the gain of the switch function, $\operatorname{sgn}(s)$, was constant.

The traditional SMC has the problem of chattering which makes the SMC method hard to apply in practice. In addition, improving the accuracy and robustness of the control system is very important for the flight of the quadrotor in complex external environment. Considering that chattering phenomenon is caused by the gain of the switch function $\operatorname{sgn}(s)$, which is generally designed to be a constant, here we propose to replace the constant with a time-varying function. The motivation of this study is to design a fuzzy SMC strategy based on logical framework with side conditions (LFSC) to allow the quadrotor to achieve an accurate trajectory without chattering. The main contributions are summarized as follows:

(1) The proposed LFSC scheme can guarantee that the tracking errors of all system state variables converge to zero in finite time.

(2) The high-frequency chattering phenomenon caused by the switching control action does not appear using the proposed LFSC scheme.

(3) Simulation results demonstrate the superior performance and robustness of the proposed LFSC scheme in the case of external disturbances.

The rest of this paper is organized as follows: in Section 2, a dynamic model of the quadrotor is presented, and the underactuated problem is solved. In Section 3, the proposed control strategy is described in detail. In Section 4, the simulation results and discussion are provided to show the superiority of the proposed control strategy. In Section 5, conclusions are presented.

\section{Model}

2.1. Description of the Quadrotor Model. A schematic diagram of a quadrotor UAV is shown in Figure 1, the earthfixed coordinate system is defined as the E-frame $\left(O_{e}, X_{e}, Y_{e}, Z_{e}\right)$, and the body-fixed system is defined as the B-frame $\left(O_{b}, X_{b}, Y_{b}, Z_{b}\right)$. The main frame of the quadrotor is assumed to be a rigid body. The four propellers are installed in two vertical directions: propellers 1 and 3 rotate in the counterclockwise direction, while propellers 2 and 4 rotate in the clockwise direction to generate a lift force and balance the yaw torque as needed. Changing all four rotor speeds by the same amount changes the lift force, thus affecting the altitude of the quadrotor. Pitch rotation can be obtained by varying the speeds of propellers 1 and 3 in opposite directions. Roll rotation can be generated in a similar way by changing the speeds of propellers 2 and 4 . The quadrotor has six degrees of freedom, including translational motions and three rotational motions, with only four independent inputs generated by increasing or decreasing the speeds of the four propellers [35]. The thrusts generated by the four rotors are denoted by $f_{i}(i=1,2,3,4)$, respectively.

2.2. Kinematic Model. As shown in Figure 1, two reference frames are defined to describe the quadrotor kinematics model: the E-frame $\left(O_{e}, X_{e}, Y_{e}, Z_{e}\right)$ and the B-frame $\left(O_{b}, X_{b}, Y_{b}, Z_{b}\right)$. In the B-frame $\left(O_{b}, X_{b}, Y_{b}, Z_{b}\right)$, this paper assumes that the origin $O_{b}$ of the body coordinate system is located at the center of the quadrotor; $X_{b}$ and $Y_{b}$ point toward rotors 1 and 2 , respectively. Then, according to the right-hand rule, $Z_{b}$ points upwards. The E-frame $\left(O_{e}, X_{e}, Y_{e}, Z_{e}\right)$ is used to define the absolute position of the quadrotor according to $X=[x, y, z]^{T}$ and Euler angles $\eta=[\phi, \theta, \psi]^{T}$, where $\phi, \theta$, and $\psi$ denote the roll angle, pitch, and yaw, respectively. $V=[\mu, v, \omega]^{T}$ and $\Omega=[p, q, r]^{T}$ denote the linear and angular velocities of the quadrotor, respectively. In this context, the quadrotor can be modelled by [36]

$$
\left\{\begin{array}{l}
\dot{X}=R_{t} V, \\
\dot{\eta}=R_{r} \Omega,
\end{array}\right.
$$

where rotation matrices are given by

$$
\begin{aligned}
R_{t} & =\left[\begin{array}{ccc}
c_{\theta} c_{\psi} & s_{\phi} s_{\theta} c_{\psi}-c_{\phi} s_{\psi} & c_{\phi} s_{\theta} c_{\psi}+s_{\phi} s_{\psi} \\
c_{\theta} s_{\psi} & s_{\phi} s_{\theta} s_{\psi}+c_{\phi} c_{\psi} & c_{\phi} s_{\theta} s_{\psi}-s_{\phi} c_{\psi} \\
-s_{\theta} & s_{\phi} c_{\theta} & c_{\phi} c_{\theta}
\end{array}\right], \\
R_{r} & =\left[\begin{array}{ccc}
1 & 0 & -s_{\theta} \\
0 & c_{\phi} & c_{\theta} s_{\phi} \\
0 & -s_{\phi} & c_{\theta} c_{\phi}
\end{array}\right],
\end{aligned}
$$




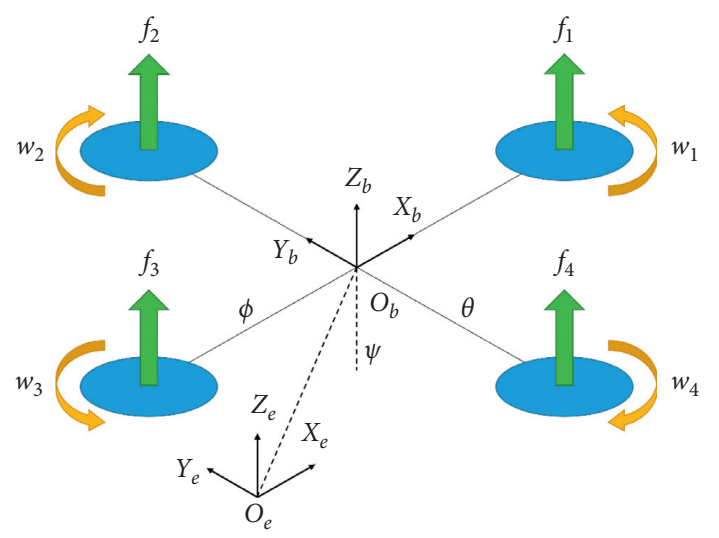

FIGURE 1: Schematic of the quadrotor.

and where $s_{*}$ and $c_{*}$ denote $\sin (*)$ and $\cos (*)$, respectively. Equations (1)-(3) are used to calculate the actual position and attitude of the quadrotor.

2.3. Dynamic Model. The dynamic model is built in consideration of model uncertainties and disturbances. It must be noted that ground and gyro effects were not taken into account because the purpose of this research was to design a control system for the model; therefore, the model was kept as simple as possible, with only the main effects being taken into account [37].

Assumption 1. The main frame of the quadrotor is symmetrical and rigid.

Assumption 2. The origin $\mathrm{O}_{b}$ of the quadrotor's body coordinate system is located at the center of mass of the quadrotor.

Assumption 3. The aerodynamic parameters of the rotors and propellers are the same.

Assumption 4. Ground and gyro effects can be ignored (in this case, compared to the brushless motor, the propeller is very light; thus, the moment of inertia due to the propeller is ignored here).

According to Newton's laws of motion and Euler's formula, a simplified dynamic model of the quadrotor is given below $[38,39]$

$$
\left\{\begin{array}{l}
m \ddot{x}=\left(c_{\phi} s_{\theta} c_{\psi}+s_{\phi} s_{\psi}\right) u_{1}-K_{1} \dot{x}, \\
m \ddot{y}=\left(c_{\phi} s_{\theta} s_{\psi}-s_{\phi} c_{\psi}\right) u_{1}-K_{2} \dot{y}, \\
m \ddot{z}=\left(c_{\phi} c_{\theta}\right) u_{1}-m g-K_{3} \dot{z}, \\
I_{x} \ddot{\phi}=u_{2}-K_{4} \dot{\phi} \\
I_{y} \ddot{\theta}=u_{3}-K_{5} \dot{\theta} \\
I_{z} \ddot{\psi}=u_{4}-K_{6} \dot{\psi},
\end{array}\right.
$$

where $m$ is the mass of the quadrotor, $g$ is the acceleration of gravity, $K_{i}(i=1,2,3,4,5,6)$ are the drag coefficients for the system, and $I_{x}, I_{y}$ and $I_{z}$ are the principal moments of inertia.

When the quadrotor is flying at low speed indoors, the control inputs $u_{1}, u_{2}, u_{3}$, and $u_{4}$ represent the lift torque, roll torque, pitch torque, and yaw torque of the quadrotor, respectively. $u_{1}, u_{2}, u_{3}$, and $u_{4}$ are as follows:

$$
\begin{aligned}
& u_{1}=\sum_{i=1}^{4} f_{i}, \\
& u_{2}=\left(f_{2}-f_{4}\right) l, \\
& u_{3}=\left(f_{3}-f_{1}\right) l, \\
& u_{4}=k_{b}\left(f_{4}-f_{3}+f_{2}-f_{1}\right),
\end{aligned}
$$

Equation (5) can be rearranged in matrix form as follows:

$$
\left[\begin{array}{l}
u_{1} \\
u_{2} \\
u_{3} \\
u_{4}
\end{array}\right]=\left[\begin{array}{cccc}
1 & 1 & 1 & 1 \\
0 & l & 0 & l \\
-l & 0 & l & 0 \\
-k_{b} & k_{b} & -k_{b} & k_{b}
\end{array}\right]\left[\begin{array}{l}
f_{1} \\
f_{2} \\
f_{3} \\
f_{4}
\end{array}\right],
$$

where $l$ is the linear distance from the center of the rotor to the center of gravity.

$$
\left\{\begin{array}{l}
f_{i}=b w_{i}^{2} \quad(i=1,2,3,4), \\
k_{b}=\frac{\alpha}{b}
\end{array}\right.
$$

where $b$ is the thrust coefficient, which depends on the blade rotor characteristics; $\alpha$ is the force to moment scaling factor [38], and $w_{i}$ is the angular speed of the $i$ th propeller of the quadrotor.

From equation (4), the quadrotor dynamics presented in E-frame in the presence of external disturbances is given by

$$
\left\{\begin{array}{l}
m \ddot{x}=\left(c_{\phi} s_{\theta} c_{\psi}+s_{\phi} s_{\psi}\right) u_{1}-K_{1} \dot{x}+d_{1}, \\
m \ddot{y}=\left(c_{\phi} s_{\theta} s_{\psi}-s_{\phi} s_{\psi}\right) u_{1}-K_{2} \dot{y}+d_{2} \\
m z=\left(c_{\phi} c_{\theta}\right) u_{1}-m g-K_{3} \dot{z}+d_{3} \\
\ddot{\phi}=\frac{1}{I_{x}} u_{2}-\frac{K_{4}}{I_{x}} \dot{\phi}+d_{4} \\
\ddot{\theta}=\frac{1}{I_{y}} u_{3}-\frac{K_{5}}{I_{y}} \dot{\theta}+d_{5} \\
\ddot{\psi}=\frac{1}{I_{z}} u_{4}-\frac{K_{6}}{I_{z}} \dot{\psi}+d_{6},
\end{array}\right.
$$

where $d_{i}(i=1,2,3,4,5,6)$ are the unknown disturbances that contain system uncertainties and other unknowns.

The control objective is to design the input control so that the quadrotor tracks the time-varying desired trajectory 
$\left[x_{d}, y_{d}, z_{d}, \phi_{d}, \theta_{d}, \psi_{d}\right]^{T}$. However, in the dynamic model of the quadrotor from equation (8), there are only four control inputs, but six outputs $x, y, z, \phi, \theta, \psi$ to control. To deal with the underactuated problem, we consider three virtual control inputs $\left(v_{1}, v_{2}, v_{3}\right)$, as follows [35]:

$$
\left\{\begin{array}{l}
v_{1}=\left(c_{\phi} s_{\theta} c_{\psi}+s_{\phi} s_{\psi}\right) u_{1}, \\
v_{2}=\left(c_{\phi} s_{\theta} s_{\psi}-s_{\phi} s_{\psi}\right) u_{1} \\
v_{3}=\left(c_{\phi} c_{\theta}\right) u_{1} .
\end{array}\right.
$$

Applying the three virtual control inputs to (8), the dynamic model can be rewritten as

$$
\left\{\begin{array}{l}
m \ddot{x}=v_{1}-K_{1} \dot{x}+d_{1}, \\
m \ddot{y}=v_{2}-K_{2} \dot{y}+d_{2}, \\
m \ddot{z}=v_{3}-m g-K_{3} \dot{z}+d_{3}, \\
I_{x} \ddot{\phi}=u_{2}-K_{4} \dot{\phi}+d_{4}, \\
I_{y} \ddot{\theta}=u_{3}-K_{5} \dot{\theta}+d_{5}, \\
I_{z} \ddot{\psi}=u_{4}-K_{6} \dot{\psi}+d_{6} .
\end{array}\right.
$$

By setting the desired yaw angle $\psi_{d}$ and using equation (9), the input control $u_{1}$, roll angle $\phi_{d}$, and pitch angle $\theta_{d}$ are given by

$$
\left\{\begin{array}{l}
u_{1}=\sqrt{v_{1}^{2}+v_{2}^{2}+v_{3}^{2}} \\
\phi_{d}=\sin ^{-1}\left(\frac{v_{1} \sin \left(\psi_{d}\right)-v_{2} \cos \left(\psi_{d}\right)}{u_{1}}\right) \\
\theta_{d}=\tan ^{-1}\left(\frac{v_{1} \cos \left(\psi_{d}\right)+v_{2} \sin \left(\psi_{d}\right)}{v_{3}}\right) .
\end{array}\right.
$$

Therefore, the trajectory tracking control objective can be described as follows: given the desired trajectory $\left[x_{d}, y_{d}, z_{d}, \psi_{d}\right]^{T}$, the idea is to design the control laws $v_{1}, v_{2}, v_{3}$ and $u_{2}, u_{3}, u_{4}$, such that the tracking errors converge to zero asymptotically.

\section{Controller Design and Stability Analysis}

In this section, the proposed controller was divided into an inner loop (attitude) controller and an outer loop (position) controller. For the inner and outer loops, a novel fuzzy sliding mode controller based on LFSC was first developed. The proposed controller guaranteed that the reference position $\left(x_{d}, y_{d}, z_{d}\right)$ and attitude $\psi_{d}$ could be accurately tracked. Using equation (10), the reference attitude $\left(\phi_{d}, \theta_{d}\right)$ could also be accurately tracked. Finally, the entire closedloop system could quickly track the reference signals. The overall control structure of the quadrotor is shown in Figure 2.

3.1. Outer Loop Controller Design. The position could be extracted from equation (10) as follows:

$$
\ddot{X}=\frac{1}{m} v-\frac{K_{o}}{m} \dot{X}-g \lambda+\frac{1}{m} d_{o},
$$

where $X=(x, y, z)^{T}, \lambda=[0,0,1]^{T}, v=\left[v_{1}, v_{2}, v_{3}\right]^{T}, K_{o}=$ $\operatorname{diag}\left(K_{1}, K_{2}, K_{3}\right)$, and $d_{o}=\left[d_{1}, d_{2}, d_{3}\right]^{T}$. Let $X_{d}=\left(x_{d}\right.$, $\left.y_{d}, z_{d}\right)^{T}$ be the desired trajectory. Defined the position tracking error as

$$
e_{X}=X_{d}-X \text {, }
$$

where $e_{X}=\left[x_{e}, y_{e}, z_{e}\right]^{T}$. Then, the LFSC manifold was given by

$$
s_{X}=c_{X} e_{X}+C \dot{e_{X}}
$$

where $c_{X}$ and $C$ were positive constants. Parameter $c_{X}$ was related to the rate of approaching the sliding mode surface, the larger the parameter $c_{X}$, the faster the approaching rate, while the greater the overshoot. Parameter $C$ is related to retain the system states on the sliding mode surface. $s_{X}=\left[s_{x}, s_{y}, s_{z}\right]^{T}$. Then, the derivative of $s_{X}$ with respect to time was

$$
\begin{aligned}
\dot{s_{X}} & =c_{X} \dot{e_{X}}+C \ddot{e_{X}} \\
& =c_{X} \dot{e_{X}}+C\left(\ddot{X_{d}}-\ddot{X}\right) \\
& =c_{X} \dot{e_{X}}+C\left(\ddot{X}_{d}-\left(\frac{1}{m} v-\frac{K_{o}}{m} \dot{X}-g \lambda+\frac{1}{m} d_{o}\right)\right) \\
& =c_{X} \dot{e_{X}}+C\left(\ddot{X}_{d}+\frac{K_{o}}{m} \dot{X}+g \lambda-\frac{1}{m} v-\frac{1}{m} d_{o}\right) .
\end{aligned}
$$

Consider the tracking error (13) and the LFSC manifold (14). The virtual control law for the outer loop was as follows:

$$
\begin{aligned}
& v_{1}=m\left(\frac{1}{C} c_{x} \dot{e}_{x}+\ddot{x}_{d}+\frac{K_{1}}{m} \dot{x}+\left|H_{1}(t)\right| \operatorname{sgn}\left(s_{x}\right)+\xi s_{x}-\frac{1}{m} \widehat{d}_{1}(t)\right), \\
& v_{2}=m\left(\frac{1}{C} c_{y} \dot{e}_{y}+\ddot{y}_{d}+\frac{K_{2}}{m} \dot{y}+\left|H_{2}(t)\right| \operatorname{sgn}\left(s_{y}\right)+\xi s_{y}-\frac{1}{m} \widehat{d}_{2}(t)\right), \\
& v_{3}=m\left(\frac{1}{C} c_{z} \dot{e}_{z}+\ddot{z_{d}}+\frac{K_{3}}{m} \dot{z}+\left|H_{3}(t)\right| \operatorname{sgn}\left(s_{z}\right)+\xi s_{z}-\frac{1}{m} \widehat{d}_{3}(t)+g\right),
\end{aligned}
$$




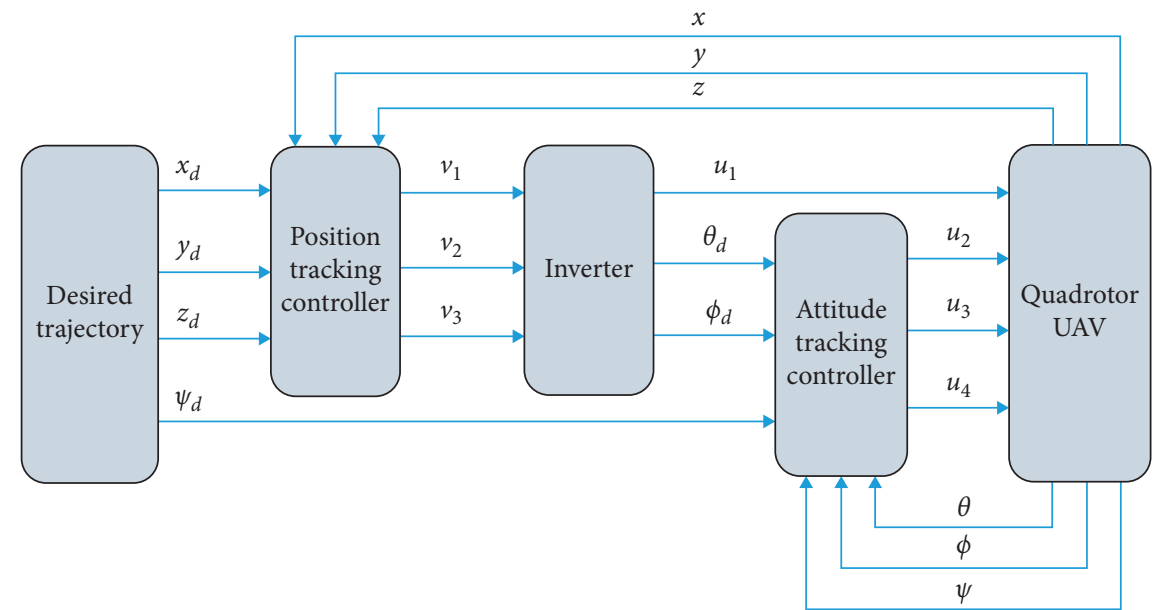

FIgURE 2: Control structure of quadrotor.

where $\xi$ was a positive constant. Parameter $\xi$ was related to retain the control system stability. $H_{1}(t), H_{2}(t)$, and $H_{3}(t)$ were the parameters obtained by the fuzzy controller. To reduce the impact of external disturbances and attenuate chattering, $d_{o}$ at the next moment must be predicted. $\widehat{d}_{o}(t)=$ $\left[\hat{d}_{1}(t), \hat{d}_{2}(t), \hat{d}_{3}(t)\right]^{T}$ was the linear prediction of disturbances $d_{o}$ and could be obtained based on the value and derivative of $d_{o}$ at the last moment:

$$
\begin{aligned}
d_{1}(t-T) & =m \ddot{x}(t-T)+K_{1} \dot{x}(t-T)-v_{1}(t-T), \\
d_{2}(t-T) & =m \ddot{y}(t-T)+K_{2} \dot{y}(t-T)-v_{2}(t-T), \\
d_{3}(t-T) & =m \ddot{z}(t-T)+K_{3} \dot{z}(t-T)-v_{3}(t-T), \\
\hat{d}_{1}(t) & =d_{1}(t-T)+\dot{d}_{1}(t-T) \times T, \\
\widehat{d}_{2}(t) & =d_{2}(t-T)+\dot{d}_{2}(t-T) \times T, \\
\widehat{d}_{3}(t) & =d_{3}(t-T)+\dot{d}_{3}(t-T) \times T,
\end{aligned}
$$

where $t$ was time and $T$ was the sampling interval of time.

$$
\operatorname{sgn}(s)= \begin{cases}1, & s>0, \\ 0, & s=0, \\ -1 & s<0 .\end{cases}
$$

The Lyapunov function was chosen as follows:

$$
V_{1}=\frac{1}{2} s_{x}^{2}
$$

Then, the derivative of $V_{1}$ with respect to time was given by

$$
\begin{aligned}
\dot{V}_{1} & =s_{x} \dot{s_{x}} \\
& =s_{x}\left(c_{x} \dot{e}_{x}+C \ddot{e}_{x}\right) \\
& =s_{x}\left(c_{x} \dot{e}_{x}+C\left(\ddot{x}_{d}+\frac{K_{1}}{m} \dot{x}-\frac{1}{m} v_{1}-\frac{1}{m} d_{1}\right)\right) .
\end{aligned}
$$

Substituting the virtual control law (16) into (20), we obtained the derivative of $V_{1}$ :

$$
\begin{aligned}
\dot{V}_{1} & =s_{x} C\left(-H_{1}(t) \operatorname{sgn}\left(s_{x}\right)-\xi s_{x}-\frac{1}{m}\left(d_{1}-\widehat{d}_{1}(t)\right)\right) \\
& =C\left(-\xi s_{x}^{2}-\left(\left|H_{1}(t)\right|\left|s_{x}\right|-\frac{1}{m}\left(d_{1}-\widehat{d}_{1}(t)\right) s_{x}\right)\right) .
\end{aligned}
$$

To guarantee $V_{1} \leq 0$, the appropriate parameters of $H_{1}(t)$ must be selected, such that $H_{1}(t)$ was sufficiently large to balance the error in the prediction of the disturbances, expressed as

$$
\left|H_{1}(t)\right| \geq \frac{1}{m}\left(d_{1}-\widehat{d}_{1}(t)\right) .
$$

The combination of (21) and (22) implied that the LFSC manifold designed in (14) was feasible. To ensure that (22) is true, a fuzzy controller could be constructed to obtain $H_{1}(t)$. The fuzzy controller in this paper has two parameters, $s_{x}, s_{x}$, as the input; $H_{1}(t)$ was the only output. The fuzzy rules are shown in Table 1 . The rules of the controllers are expressed in Table 1, for all possible combinations. Based on the 
TABLe 1: Fuzzy rules.

\begin{tabular}{|c|c|c|c|c|c|c|c|c|}
\hline & \multirow{2}{*}{$H_{1}(t)$} & \multicolumn{7}{|c|}{$s_{x}$} \\
\hline & & NB & NM & NS & $\mathrm{ZO}$ & PS & PM & PB \\
\hline \multirow{7}{*}{$s_{x}$} & $\mathrm{~PB}$ & NS & PS & PM & PM & $\mathrm{PM}$ & $\mathrm{PB}$ & PB \\
\hline & $\mathrm{PM}$ & NS & NS & PS & PS & $\mathrm{PM}$ & $\mathrm{PB}$ & PB \\
\hline & PS & NM & NS & $\mathrm{ZO}$ & $\mathrm{ZO}$ & PS & PM & PB \\
\hline & $\mathrm{ZO}$ & NM & NS & $\mathrm{ZO}$ & $\mathrm{ZO}$ & $\mathrm{ZO}$ & PS & $\mathrm{PM}$ \\
\hline & NS & NM & NM & NS & $\mathrm{ZO}$ & $\mathrm{ZO}$ & PS & $\mathrm{PM}$ \\
\hline & NM & NM & $\mathrm{NM}$ & NM & NS & NS & NM & PS \\
\hline & $\mathrm{NB}$ & NB & NB & NB & NM & NM & NB & NB \\
\hline
\end{tabular}

PB: positive big, PM: positive middle, PS: positive small, NB: negative big, NM: negative middle, NS: negative small, and ZO: zero.

control experience of the quadrotor, the fuzzy rules were established according to $s_{x}$ and $\dot{s}_{x}$ to adjust parameter $H_{1}(t)$. The membership function of the fuzzy controller is shown in Figure 3.

From Table 1, there were two cases: (i) When $s_{x} \dot{s}_{x} \leq 0$, the Lyapunov function $V_{1} \leq 0$ and parameter $H_{1}(t)$ would decrease to attenuate chattering in the control input. (ii) When $s_{x} s_{x}>0$, parameter $H_{1}(t)$ could not eliminate the effects of disturbance $d_{o}$. Thus, $H_{1}(t)$ would increase to the point of eliminating the effect of disturbance $d_{1}$, such that $s_{x} s_{x} \leq 0$ and the Lyapunov function $V_{1} \leq 0$. Based on the Lyapunov method, the system was asymptotically stable:

$$
\begin{aligned}
\dot{V_{2}} & =s_{y} \dot{s}_{y} \\
& =s_{y}\left(c_{y} \dot{e}_{y}+C \ddot{e}_{y}\right) \\
& =s_{y}\left(c_{y} \dot{e}_{y}+C\left(\ddot{y}_{d}+\frac{K_{2}}{m} \dot{y}-\frac{1}{m} v_{2}-\frac{1}{m} d_{2}\right)\right) \\
& =s_{y} C\left(-H_{2}(t) \operatorname{sgn}\left(s_{y}\right)-\xi s_{y}-\frac{1}{m}\left(d_{2}-\widehat{d}_{2}(t)\right)\right) \\
& =C\left(-\xi s_{y}^{2}-\left(\left|H_{2}(t)\right|\left|s_{y}\right|-\frac{1}{m}\left(d_{2}-\widehat{d}_{2}(t)\right) s_{y}\right)\right), \\
\dot{V} & =s_{z} \dot{s}_{z} \\
& =s_{z}\left(c_{z} \dot{e}_{z}+C \ddot{e}_{z}\right) \\
& =s_{z}\left(c_{z} \dot{e}_{z}+C\left(\ddot{z_{d}}+\frac{K_{3}}{m} \dot{z}-\frac{1}{m} v_{3}-\frac{1}{m} d_{3}\right)\right) \\
& =s_{z} C\left(-H_{3}(t) \operatorname{sgn}\left(s_{z}\right)-\xi s_{z}-\frac{1}{m}\left(d_{3}-\widehat{d}_{3}(t)\right)\right) \\
& =C\left(-\xi s_{z}^{2}-\left(\left|H_{3}(t)\right|\left|s_{z}\right|-\frac{1}{m}\left(d_{3}-\widehat{d}_{3}(t)\right) s_{z}\right)\right) .
\end{aligned}
$$

$$
\begin{aligned}
& u_{2}=J\left(\frac{1}{C_{1}} c_{\phi} \dot{e}_{\phi}+\ddot{\phi}_{d}+\frac{K_{4}}{J} \dot{\phi}+H_{4}(t) \operatorname{sgn}\left(s_{\phi}\right)+\xi_{1} s_{\phi}-\hat{d}_{4}(t)\right), \\
& u_{3}=J\left(\frac{1}{C_{1}} c_{\theta} \dot{e}_{\theta}+\ddot{\theta}_{d}+\frac{K_{5}}{J} \dot{\theta}+H_{5}(t) \operatorname{sgn}\left(s_{\theta}\right)+\xi_{1} s_{\theta}-\widehat{d}_{5}(t)\right), \\
& u_{4}=J\left(\frac{1}{C_{1}} c_{\psi} \dot{e}_{\psi}+\ddot{\psi}_{d}+\frac{K_{6}}{J} \dot{\psi}+H_{6}(t) \operatorname{sgn}\left(s_{\psi}\right)+\xi_{1} s_{\psi}-\hat{d}_{6}(t)\right) .
\end{aligned}
$$




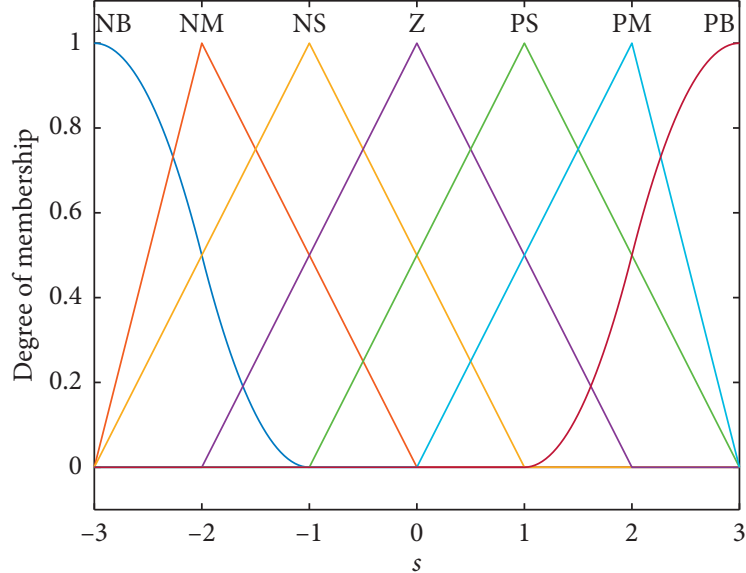

(a)

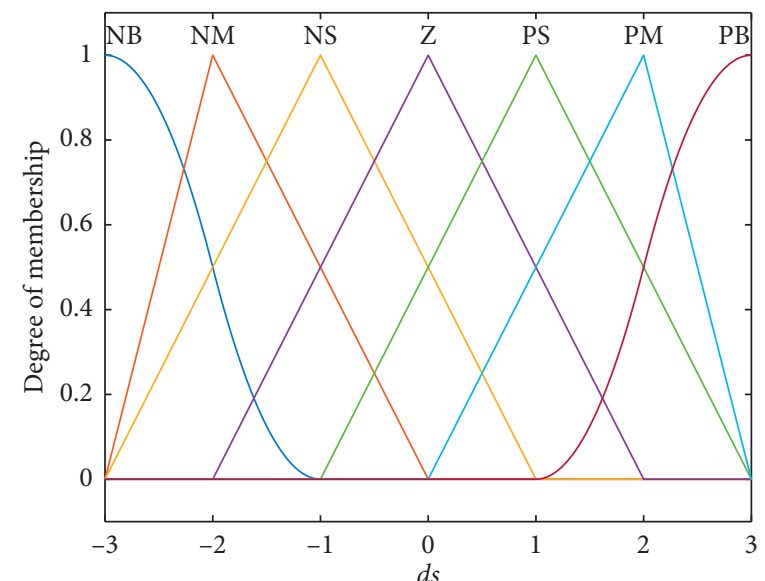

(b)

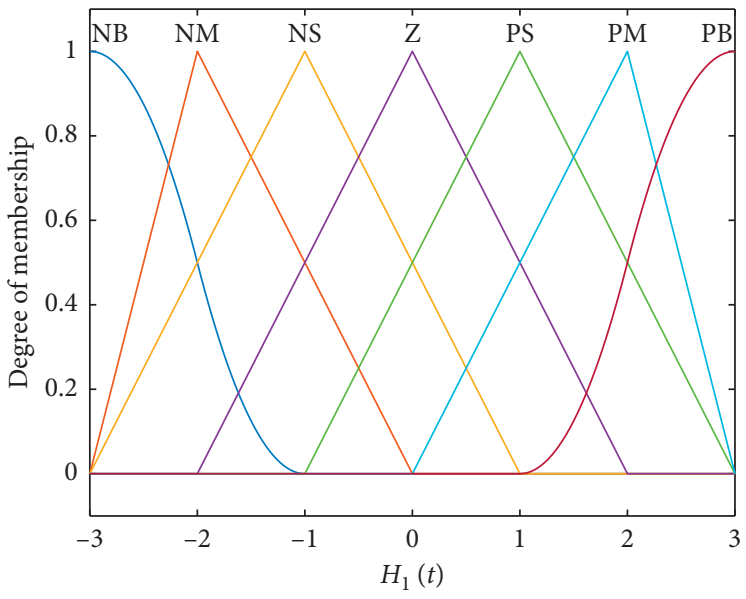

(c)

FIgURE 3: Fuzzy membership functions.

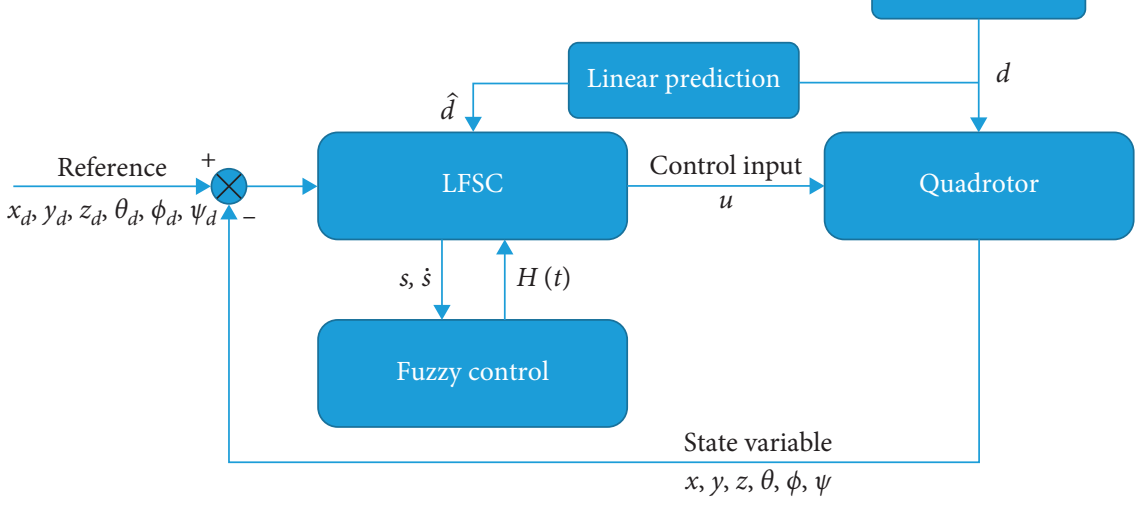

FIgURE 4: Block diagram of the LFSC controller.

where $\xi_{1}$ was a positive constant, the meaning of $\xi_{1}$ was similar to $\xi$. Then, the meaning of $H_{4}(t), H_{5}(t), H_{6}(t)$ and $\widehat{d}_{4}(t), \hat{d}_{5}(t), \widehat{d}_{6}(t)$ were similar to $H_{1}(t)$ and $\widehat{d}_{1}(t)$ in Section 3.1 , respectively.
The Lyapunov function was as follows:

$$
V_{4}=\frac{1}{2} s_{\phi}^{2}
$$


The derivative of $V_{4}$ with respect to time was given by

$$
\begin{aligned}
\dot{V}_{4} & =s_{\phi} \dot{s_{\phi}} \\
& =s_{\phi}\left(c_{\phi} \dot{e_{\phi}}+C_{1} \ddot{e_{\phi}}\right) \\
& =s_{\phi}\left(c_{\phi} \dot{e_{\phi}}+C_{1}\left(\ddot{\phi_{d}}-\frac{1}{J} u_{2}+\frac{K_{4}}{J} \dot{\phi}-d_{4}\right)\right) .
\end{aligned}
$$

Substitute the control law (28) into (30). Then, the derivative of $V_{4}$ was given by

$$
\begin{aligned}
\dot{V}_{4} & =s_{\phi}\left(-\left|H_{4}(t)\right| \operatorname{sgn}\left(s_{\phi}\right)-\xi_{1} s_{\phi}-\left(d_{4}-\hat{d}_{4}(t)\right)\right) \\
& =-\xi_{1} s_{\phi}^{2}-\left(\left|H_{4}(t)\right|\left|s_{\phi}\right|-\left(d_{4}-\widehat{d}_{4}(t)\right) s_{\phi}\right) .
\end{aligned}
$$

To guarantee $V_{4} \leq 0$, appropriate parameters of $H_{4}(t)$ must be selected, such that $\left|H_{4}(t)\right|$ was sufficient to balance the error in the prediction of interference, which could be expressed as

$$
\left|H_{4}(t)\right| \geq\left(d_{4}-\widehat{d}_{4}(t)\right)
$$

The combination of (31) and (32) implied that the sliding manifold design described in (26) was feasible.

The proof process of equation (32) was similar to equation (22) in Section 3.1, in which $s_{\phi} \dot{s}_{\phi} \leq 0$, and the Lyapunov function $V_{4} \leq 0$ could be guaranteed. Based on the Lyapunov method, the system was asymptotically stable:

$$
\begin{aligned}
& \dot{V}_{5}=s_{\theta} \dot{s_{\theta}} \\
& =s_{\theta}\left(c_{\theta} \dot{e}_{\theta}+C_{1} \ddot{e}_{\theta}\right) \\
& =s_{\theta}\left(c_{\theta} \dot{e}_{\theta}+C_{1}\left(\ddot{\theta_{d}}-\frac{1}{J} u_{3}+\frac{K_{5}}{J} \dot{\theta}-d_{5}\right)\right) \\
& =s_{\theta}\left(-\left|H_{5}(t)\right| \operatorname{sgn}\left(s_{\theta}\right)-\xi_{1} s_{\theta}-\left(d_{5}-\widehat{d}_{5}(t)\right)\right) \\
& =-\xi_{1} s_{\theta}^{2}-\left(\left|H_{5}(t)\right|\left|s_{\theta}\right|-\left(d_{5}-\widehat{d}_{5}(t)\right) s_{\theta}\right), \\
& \dot{V}_{6}=s_{\psi} \dot{s}_{\psi} \\
& \begin{array}{l}
=s_{\psi}\left(c_{\psi} \dot{e}_{\psi}+C_{1} \ddot{e}_{\psi}\right) \\
=s_{\psi}\left(c_{\psi} \dot{e}_{\psi}+C_{1}\left(\ddot{\psi}_{d}-\frac{1}{J} u_{4}+\frac{K_{6}}{J} \dot{\psi}-d_{6}\right)\right) \\
=s_{\psi}\left(-\left|H_{6}(t)\right| \operatorname{sgn}\left(s_{\psi}\right)-\xi_{1} s_{\psi}-\left(d_{6}-\widehat{d}_{6}(t)\right)\right) \\
=-\xi_{1} s_{\psi}^{2}-\left(\left|H_{6}(t)\right|\left|s_{\psi}\right|-\left(d_{6}-\widehat{d}_{6}(t)\right) s_{\psi}\right) .
\end{array}
\end{aligned}
$$

The proof process of $\dot{V}_{5} \leq 0$ and $\dot{V}_{6} \leq 0$ were similar to that of $V_{4} \leq 0$.

\section{Results and Discussion}

In this section, several trajectory tracking simulation experiments were performed in the MATLAB R2016b/Simulink, which was equipped in a computer consisting of a $2.60 \mathrm{GHz}$ CPU with $8 \mathrm{~GB}$ of RAM and a $256 \mathrm{~GB}$ solid-state disk drive. The control performance obtained by the proposed LFSC scheme was compared to SMC [40] and fuzzy SMC [41] schemes, to demonstrate the superiority of the proposed LFSC strategy.

The parameters of the quadrotor used in the simulation studies are shown in Table 2. The external disturbances considered in all of the simulation studies, to validate the robustness of the proposed LFSC control strategy, were time-varying.

Case 1. In this case, the desired trajectory of the position and yaw angle was given by

$$
\left\{\begin{array}{l}
x_{d}=\sin (t)+2 \\
y_{d}=\cos (t)+2 \\
z_{d}=0.5 t+1 \\
\psi_{d}=0
\end{array}\right.
$$

The initial position and yaw angle of the quadrotor $\left[x_{0}, y_{0}, z_{0}, \psi_{0}\right]$ were $[1.9,2.9,0.5,0.001]$. The Gaussian function and white noise functions which were imposed on the quadrotor were given by

$$
d_{i}=\left\{\begin{array}{l}
M \cos (t)+12 \exp \left(-\frac{1}{2} \times\left(\frac{t-15}{0.1}\right)^{2}\right) \quad(i=1,2,3), \\
M_{1} \cos (t) \quad(i=4,5,6) .
\end{array}\right.
$$

In order to achieve appropriate control performance, appropriate parameters $c_{X}, \xi, C, c_{\eta}, \xi_{1}$, and $C_{1}$ were designed according to reference [38]. The parameters for the proposed LFSC controllers were shown in Table 3.

Simulation results are shown in Figures 5-11. To demonstrate the superiority of the proposed LFSC scheme, simulation experiments of traditional SMC and fuzzy SMC were conducted, more details of the SMC and the fuzzy SMC methods for a quadrotor UAV have been introduced in $[40,41]$, respectively. The trajectory tracking results in $3 \mathrm{D}$ space are shown in Figure 5. The position tracking errors $\left(e_{x}, e_{y}, e_{z}\right)$ are shown in Figure 6. The following results can be observed from Figure 6: (1) It could be seen that starting from an initial position far from the desired trajectory, the proposed LFSC method successfully forces state variables $(x, y, z)$ to their desired trajectory. (2) The proposed LFSC method successfully forces position tracking errors $\left(e_{x}, e_{y}, e_{z}\right)$ to converge to zero in finite time, while the SMC and fuzzy SMC methods force position tracking errors $\left(e_{x}, e_{y}, e_{z}\right)$ to converge to small bounded fields around zero. (3) Moreover, when disturbed by the wind gust, time is about $15 \mathrm{~s}$, the performance of the proposed LFSC controller was much better than that achieved by SMC or fuzzy SMC 
TABle 2: Parameters of the model.

\begin{tabular}{lccc}
\hline Parameter & Description & Value & \\
\hline$M$ & Quadrotor mass & 2 & $\mathrm{Unit}$ \\
$L$ & Plane arm & 0.25 & $\mathrm{~kg}$ \\
$K_{i}(i=1,2,3)$ & Disturb value & 0.01 & $\mathrm{~m}$ \\
$K_{i}(i=4,5,6)$ & Disturb value & 0.001 & $\mathrm{~N} / \mathrm{m}$ \\
$G$ & Gravitational acceleration & 9.81 & $\mathrm{~N} \cdot \mathrm{m} \cdot \mathrm{s} / \mathrm{rad}$ \\
$I_{x}$ & Moment of inertia about $X$-axis & $\mathrm{m} / \mathrm{s}^{2}$ & $\mathrm{~kg} \cdot \mathrm{m} \mathrm{m}^{2}$ \\
$I_{y}$ & Moment of inertia about $Y$-axis & $8 \times 10^{-3}$ & $\mathrm{~kg} \cdot \mathrm{m}^{2}$ \\
$I_{z}$ & Moment of inertia about $Z$-axis & $8 \times 10^{-3}$ & $\mathrm{~kg} \cdot \mathrm{m}^{2}$ \\
\hline
\end{tabular}

controllers in terms of settle time, overshoot, and robustness. It was clearly seen that the proposed LFSC method was able to make the quadrotor follow the desired position trajectory with strong robustness and the highest accuracy. Due to the linear prediction and the fuzzy controller, disturbances were well compensated.

The attitude tracking errors $\left(e_{\phi}, e_{\theta}, e_{\psi}\right)$ are shown in Figure 7. The following results can be observed from Figure 7: (1) The proposed LFSC method successfully forces attitude tracking errors $\left(e_{\phi}, e_{\theta}, e_{\psi}\right)$ to converge to zero in finite time, while the SMC and fuzzy SMC methods force attitude tracking errors $\left(e_{\phi}, e_{\theta}, e_{\psi}\right)$ to converge to small bounded fields around zero. (2) The performance of the proposed LFSC method was much better than that achieved by the SMC or fuzzy SMC method in terms of tracking accuracy and robustness. It could be seen that the timevariant disturbances were not well compensated with traditional SMC or fuzzy SMC method. By introducing the linear prediction and new fuzzy controller, the LFSC method achieved good attitude tracking performance.

The response curves of virtual control input $v_{1}, v_{2}$, and $v_{3}$ under the three control methods are displayed in Figures 8-10, respectively. By observing these figures, the high-frequency chattering phenomenon caused by the switching control action are shown in Figures 8 and 9. While in Figure 10, chattering was considerably reduced by adopting the proposed LFSC control law. In Figure 10, the changes in three LFSC virtual control inputs $v_{1}, v_{2}, v_{3}$ were shown, whereby the virtual control input $v_{3}$ was around 19.6 N, which was equal to the gravity force of the quadrotor. In addition, three virtual control inputs $v_{1}, v_{2}, v_{3}$ showed huge fluctuations around $15 \mathrm{~s}$, which is caused by the wind gust.

In Figure 11, the changes in four control inputs $\left(u_{1}, u_{2}, u_{3}, u_{4}\right)$ are shown, whereby the control input $u_{1}$ was 19.71 N, which was slightly greater than the gravity force of the quadrotor. Compared with the results given in $[40,41]$, the amplitudes of the proposed LFSC controllers $\left(u_{1}, u_{2}, u_{3}, u_{4}\right)$ were greatly decreased in the presence of external disturbances.

Case 2. In order to further evaluate the performance of the proposed LFSC control strategy, the disturbances were simulated by white noise functions with standard deviation of 2 . In this case, the desired trajectory of the position and yaw angle was given by
TABLE 3: Parameters of controllers.

\begin{tabular}{lc}
\hline Parameter & Unit \\
\hline$c_{X}$ & 15 \\
$M$ & 0.5 \\
$\xi$ & 17 \\
$C$ & 1 \\
$c_{\eta}$ & 20 \\
$M_{1}$ & 0.2 \\
$\xi_{1}$ & 15 \\
$C_{1}$ & 1 \\
\hline
\end{tabular}

$$
\left\{\begin{array}{l}
x_{d}=5 \sin (0.4 t), \\
y_{d}=5 \cos (0.4 t), \\
z_{d}=3-2 \cos (0.4 t), \\
\psi_{d}=0 .
\end{array}\right.
$$

The initial position and yaw angle of the quadrotor $\left[x_{0}, y_{0}, z_{0}, \psi_{0}\right]$ were $[0.5,4.5,0.5,0.5]$. The disturbances which were imposed on the quadrotor were given by

$$
\begin{aligned}
& d_{i}=\left\{\begin{array}{ll}
2 \sin (2 t+5) & (t \leq 15 \mathrm{~s}) \\
25 & (\text { else })
\end{array} \quad(\mathrm{N}) \quad(i=1,2,3)\right. \\
& d_{i}=\left\{\begin{array}{ll}
2 \sin (1.5 t+5) & (t \leq 15 \mathrm{~s}) \\
30 & (\text { else })
\end{array}(\mathrm{N} \times \mathrm{m}) \quad(i=4,5,6)\right.
\end{aligned}
$$

Simulation results are shown in Figures 12-18. The trajectory tracking results in $3 \mathrm{D}$ space are shown in Figure 12 . The position tracking errors $\left(e_{x}, e_{y}, e_{z}\right)$ are shown in Figure 13. When the disturbance $d_{i}$ is introduced at $t=15 \mathrm{~s}$, the null steady-state error could not be reached with both SMC method and fuzzy SMC method. In contrast, the proposed LFSC control strategy is able to force the quadrotor follow the desired trajectory with the highest accuracy and null steady-state error. Due to the linear prediction and the fuzzy controller, disturbances were well compensated.

The attitude tracking errors $\left(e_{\phi}, e_{\theta}, e_{\psi}\right)$ are shown in Figure 14. It could be seen that the time-variant disturbances were not well compensated with both SMC method and fuzzy SMC method when the time was up to 15 seconds. By introducing the linear prediction and the fuzzy controller, the LFSC method achieves good attitude tracking performance. 


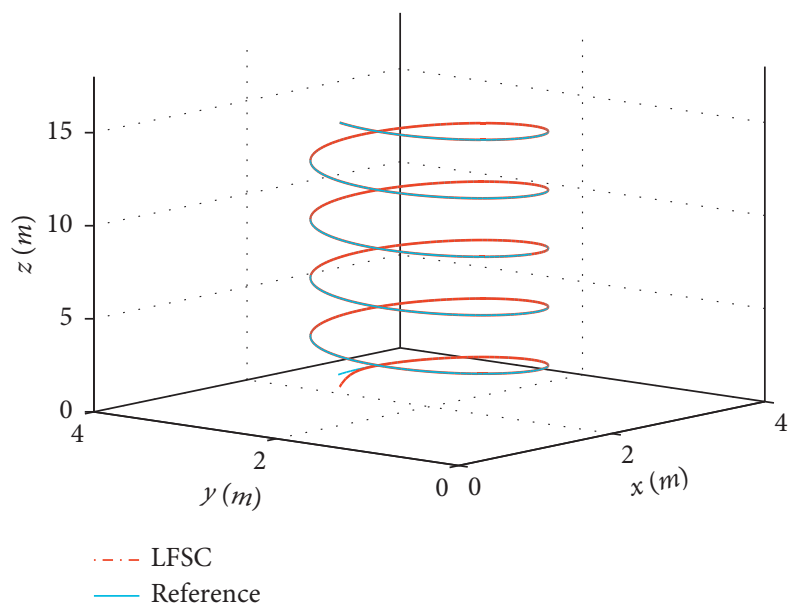

FIGURE 5: Space diagram of position.

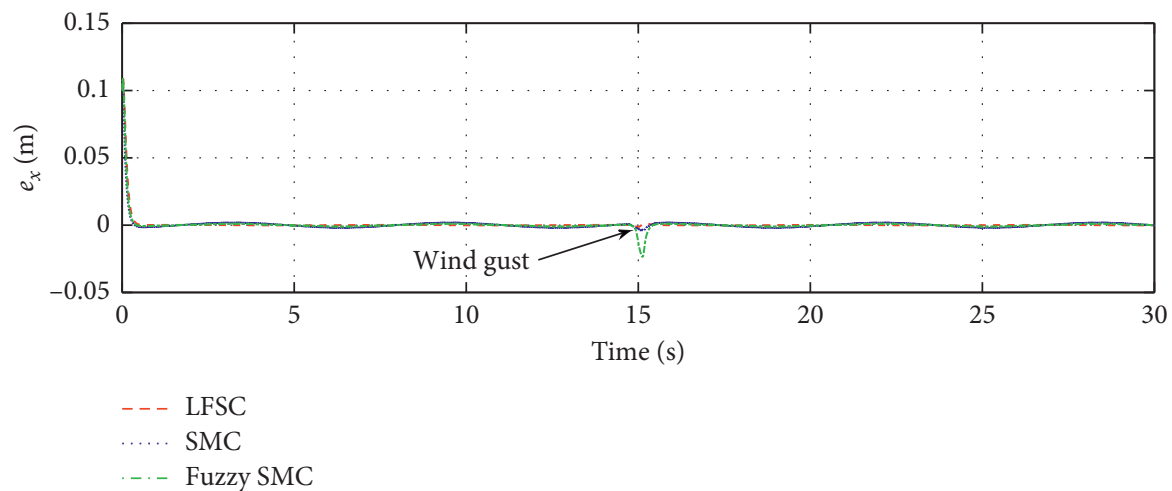

(a)

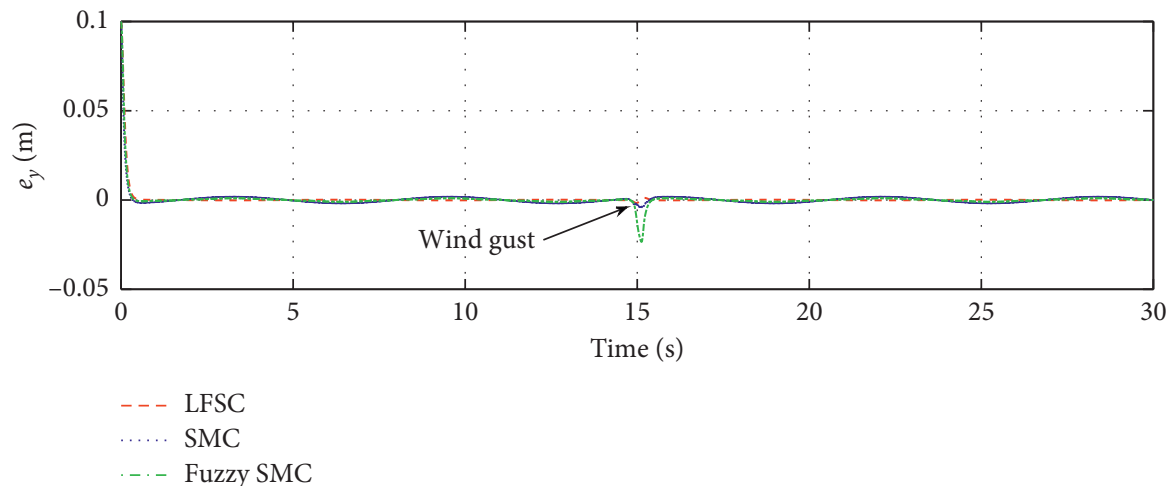

(b)

Figure 6: Continued. 


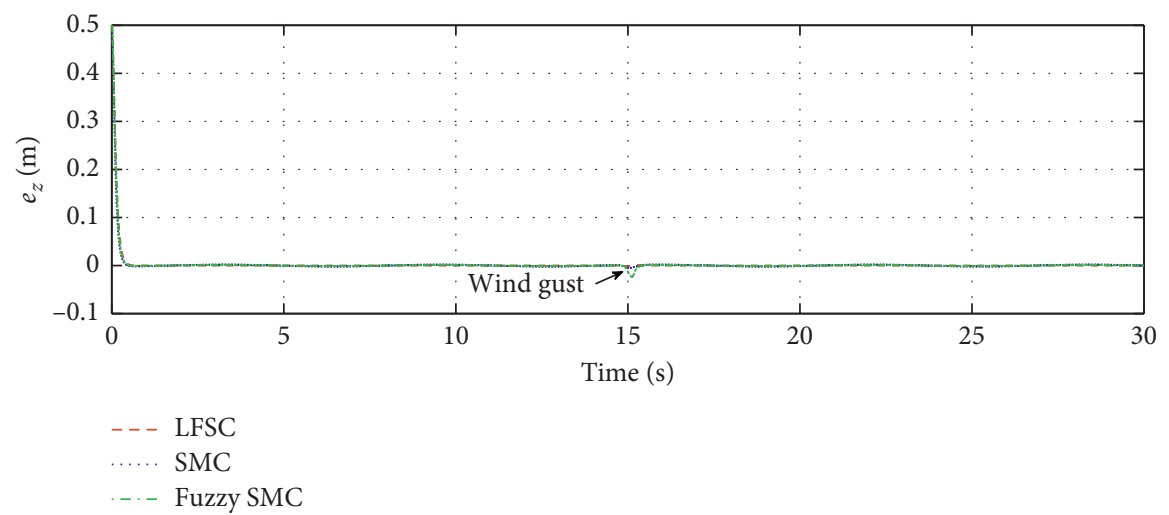

(c)

FIgURE 6: Trajectory tracking errors $\left(e_{x}, e_{y}, e_{z}\right)$.

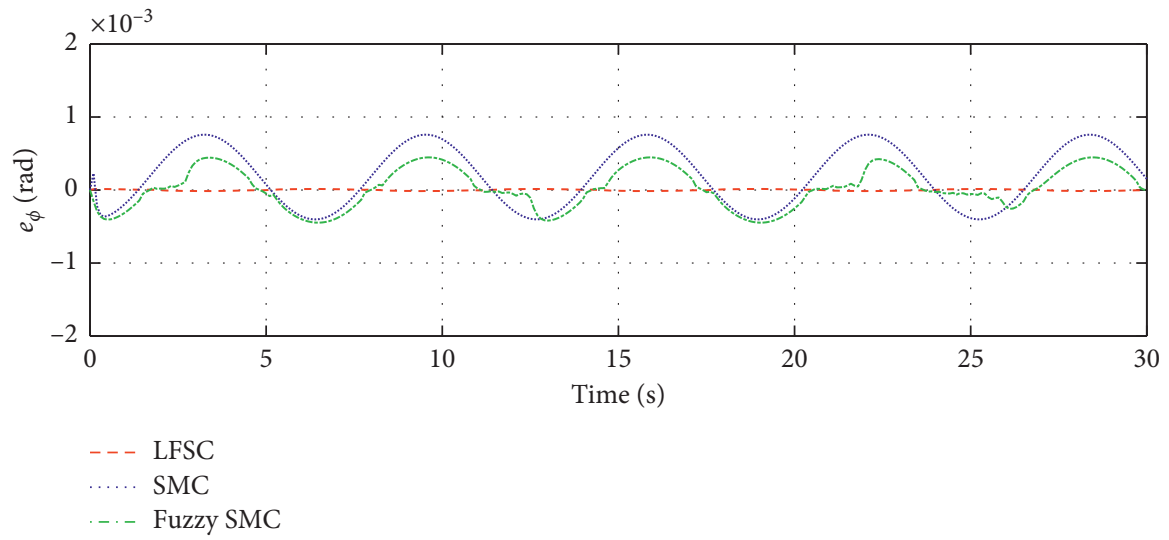

(a)

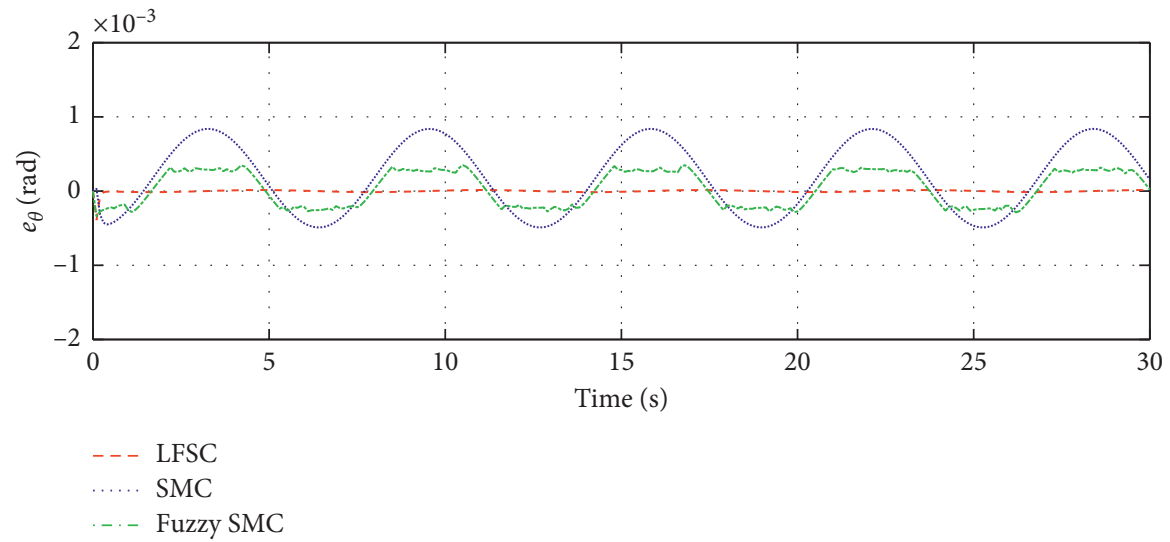

(b)

FIgURE 7: Continued. 


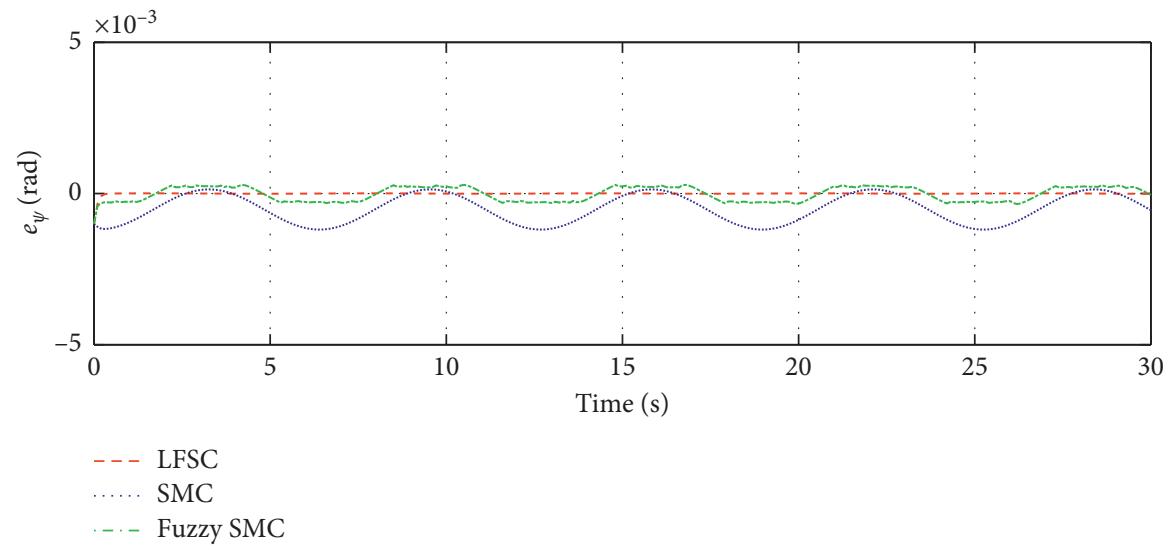

(c)

Figure 7: Trajectory tracking errors $\left(e_{\phi}, e_{\theta}, e_{\psi}\right)$.

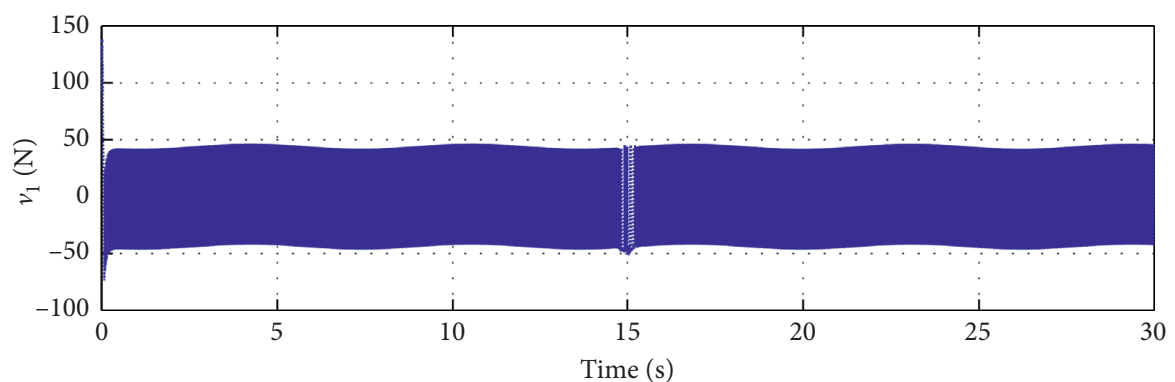

(a)

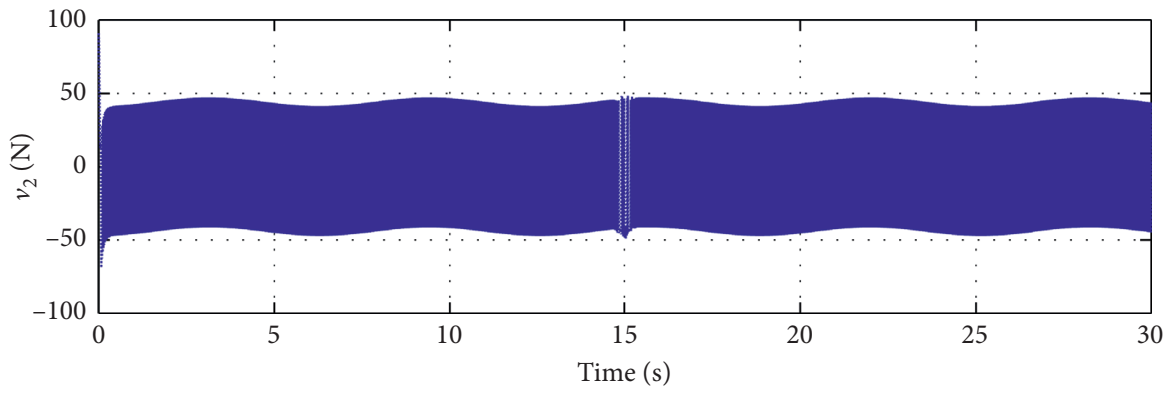

(b)

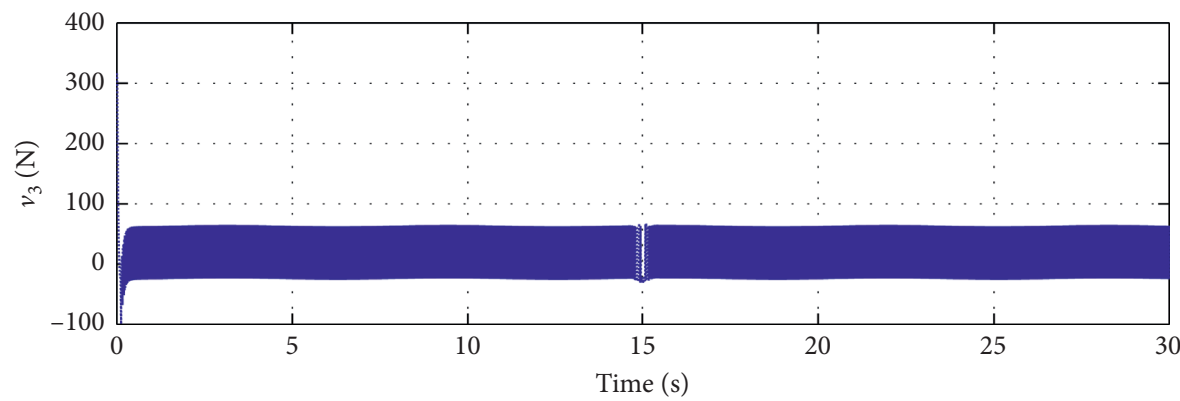

(c)

Figure 8: Virtual control input $v_{1}, v_{2}$, and $v_{3}$ of SMC.

The response curves of virtual control input $v_{1}, v_{2}$, and $v_{3}$ under the three control methods are displayed in Figures 15-18, respectively. By observing these figures, the high-frequency chattering phenomenon caused by the switching control action are shown in Figures 15 and 16. While in Figures 17 and 18, due to the linear prediction and the fuzzy controller, the chattering problems was considerably reduced by adopting the proposed LFSC control law. 


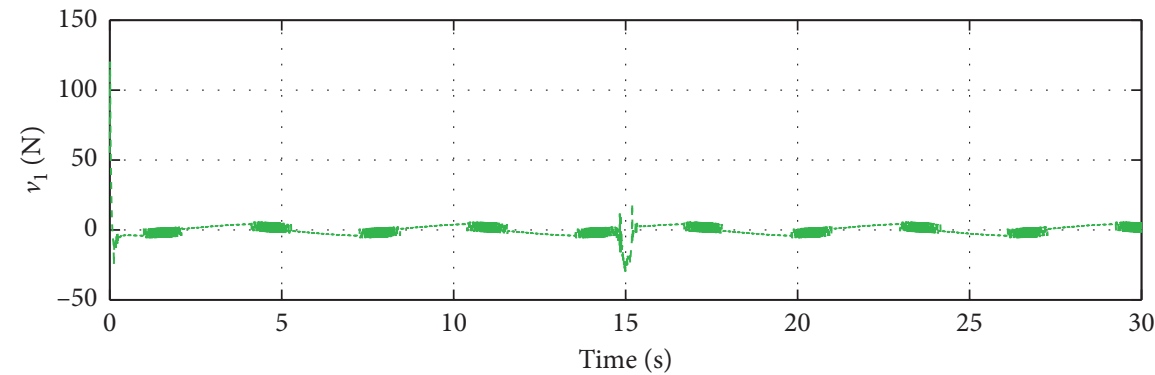

(a)

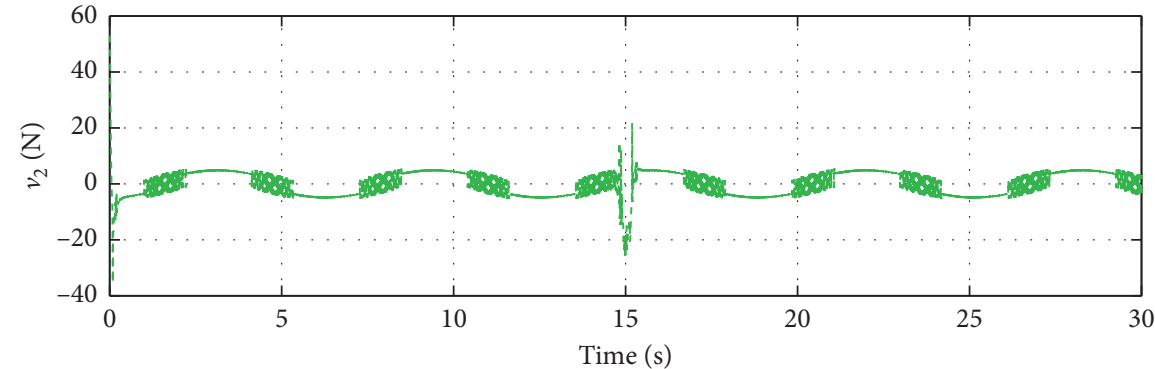

(b)

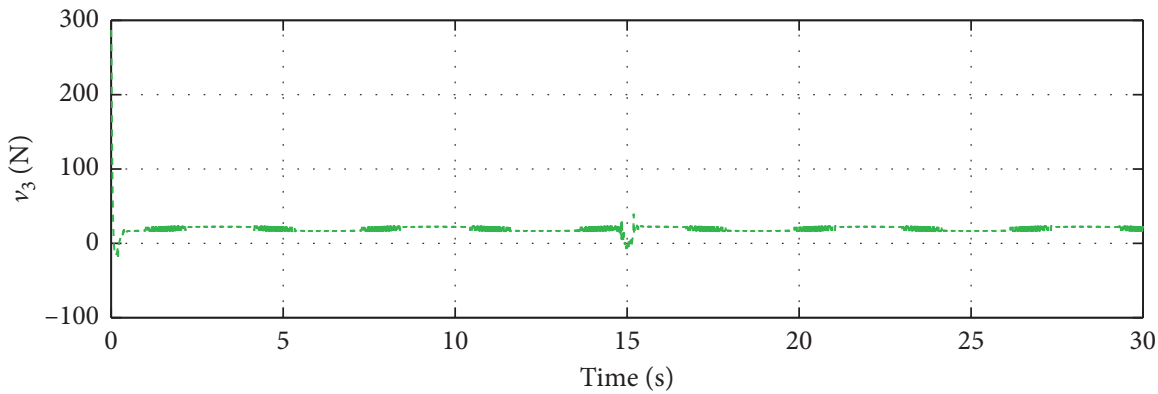

(c)

FIgURE 9: Virtual control input $v_{1}, v_{2}$, and $v_{3}$ of FSMC.

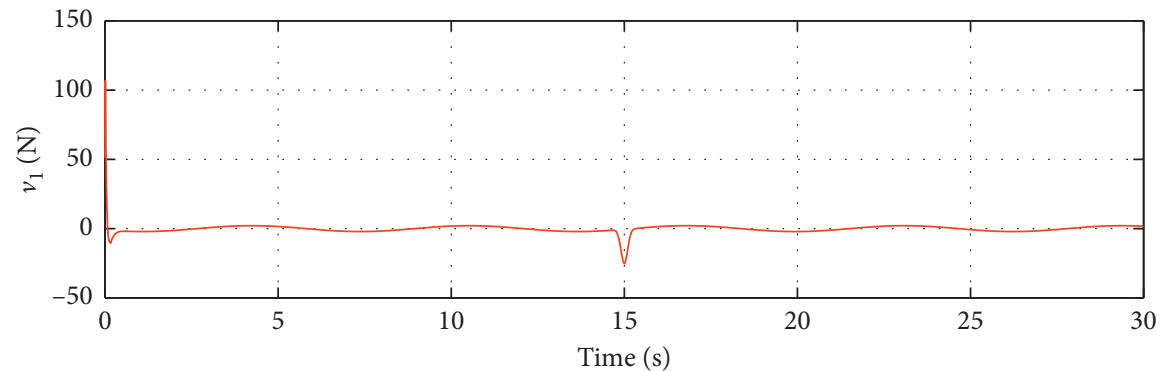

(a)

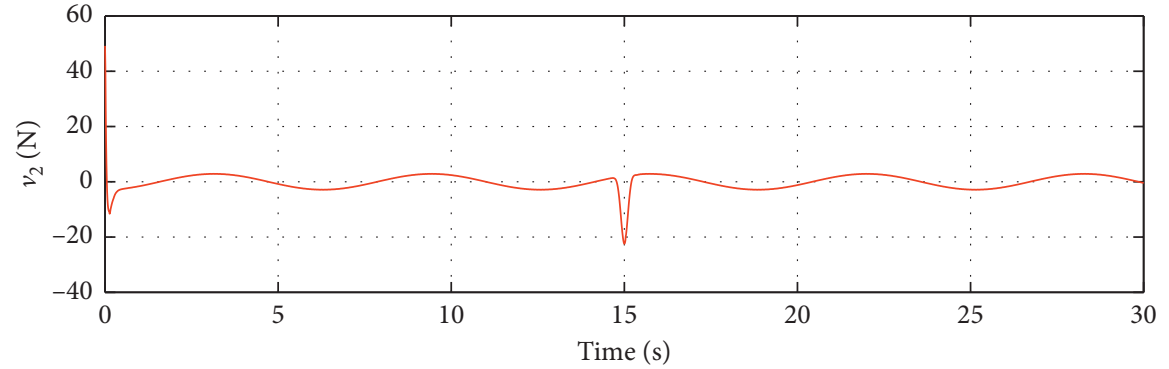

(b)

FIgURE 10: Continued. 


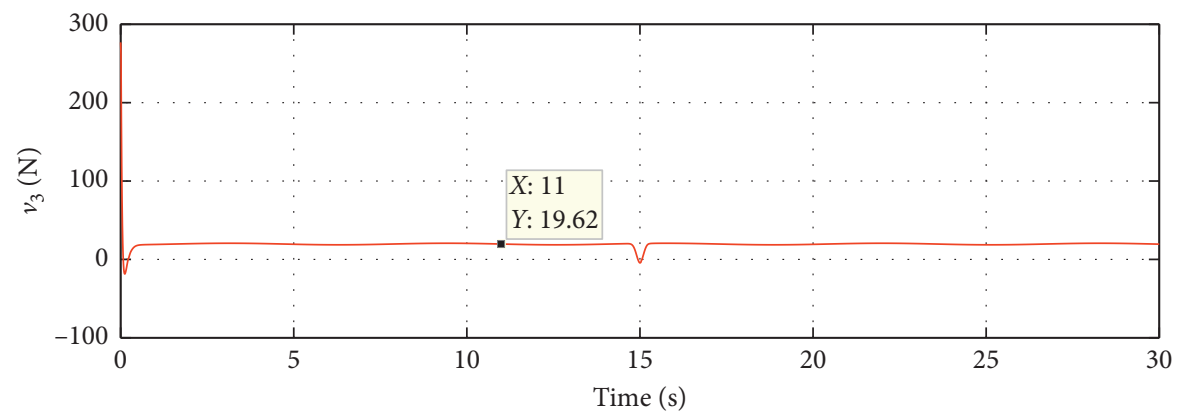

(c)

Figure 10: Virtual control input $v_{1}, v_{2}$, and $v_{3}$ of LFSC.

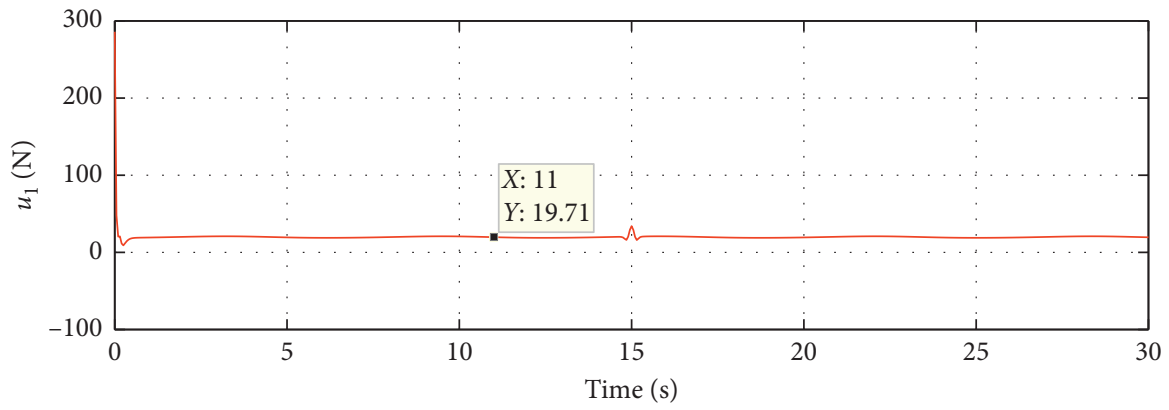

(a)

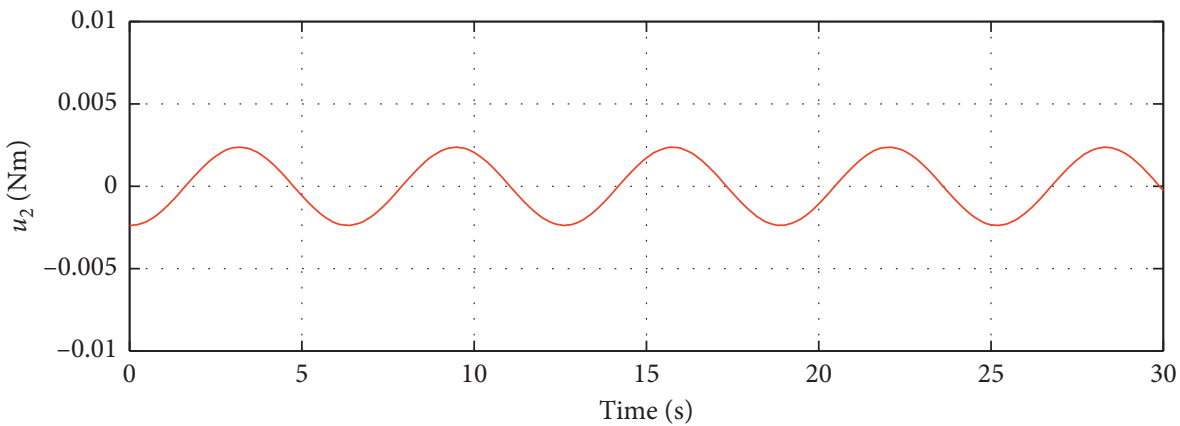

(b)

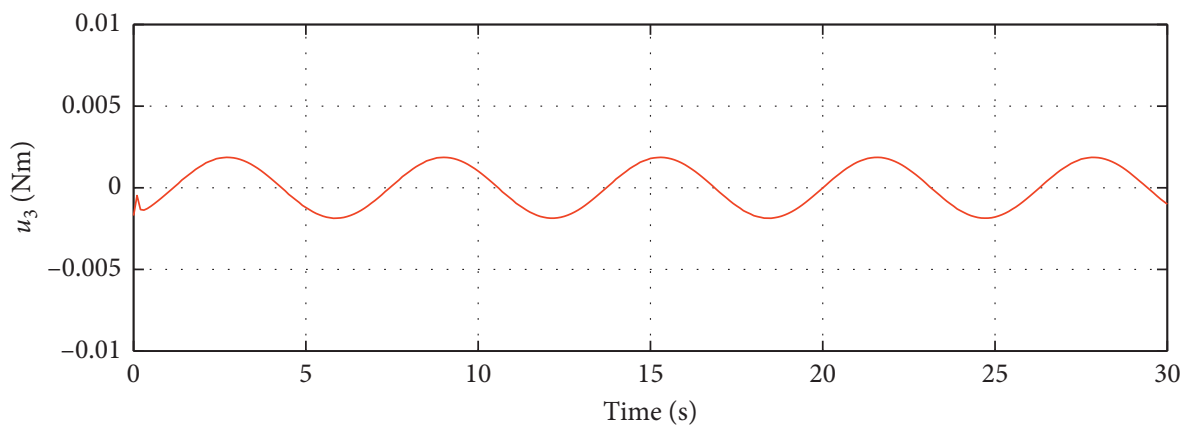

(c)

FIgURE 11: Continued. 


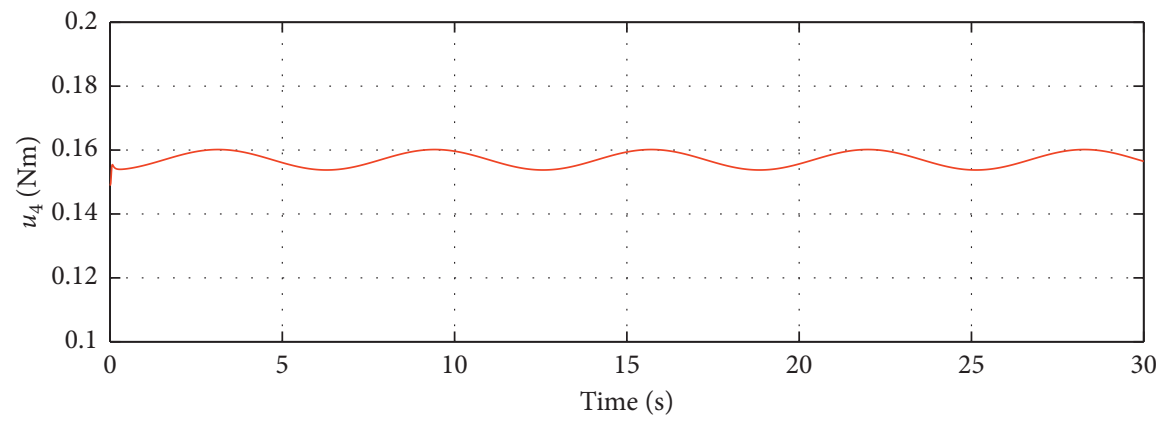

(d)

Figure 11: Control inputs $u_{1}, u_{2}, u_{3}$, and $u_{4}$ of LFSC.

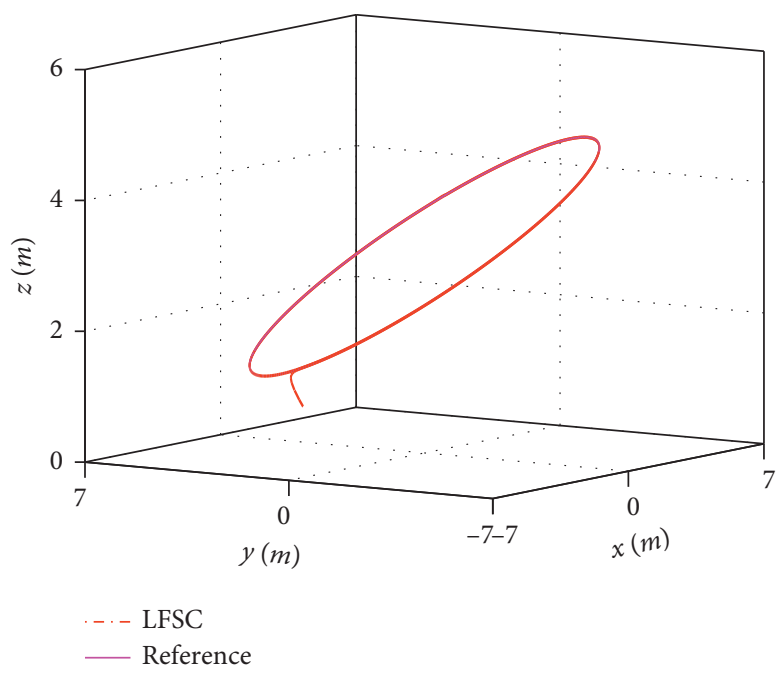

FIGURE 12: Space diagram of position.

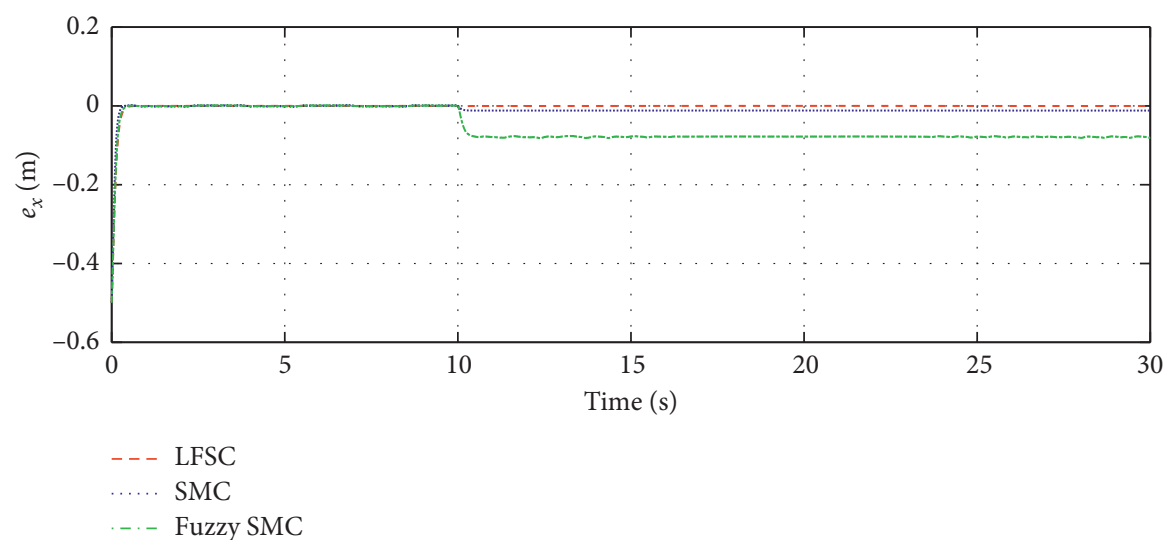

(a)

FIgURE 13: Continued. 


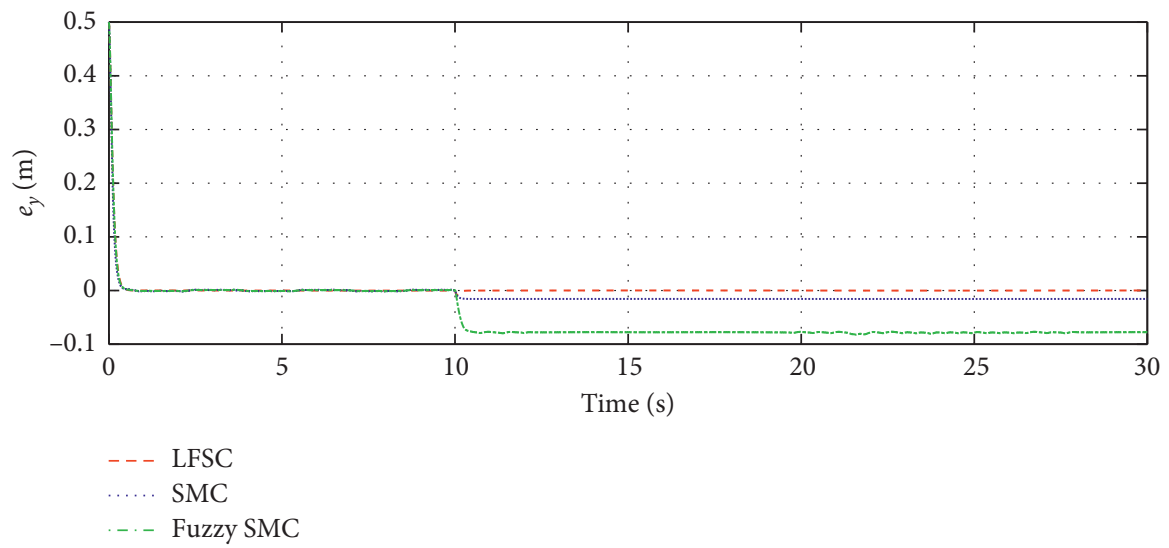

(b)

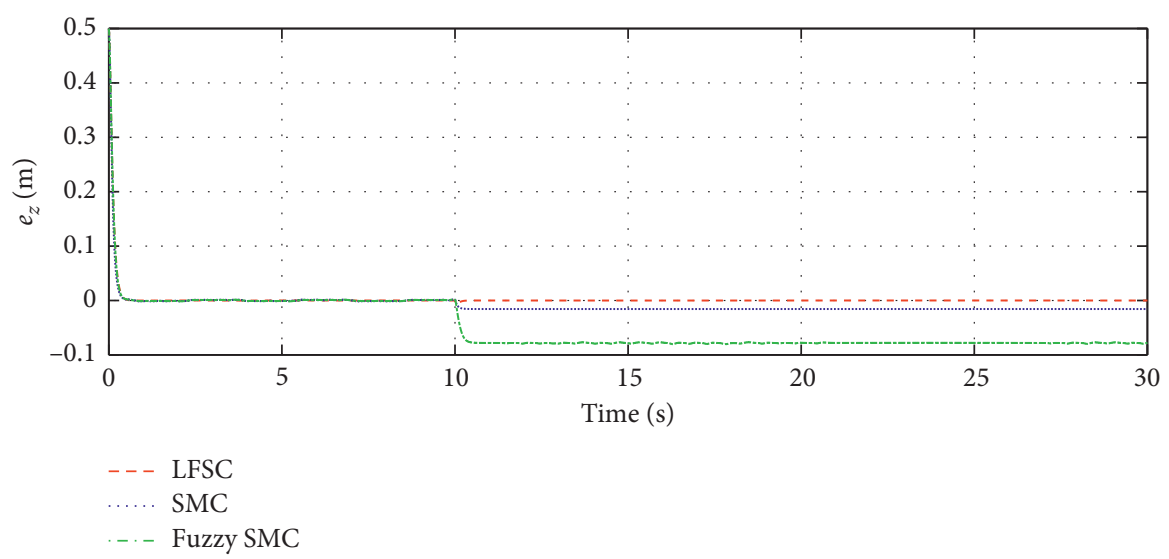

(c)

FIGURE 13: Trajectory tracking errors $\left(e_{x}, e_{y}, e_{z}\right)$.

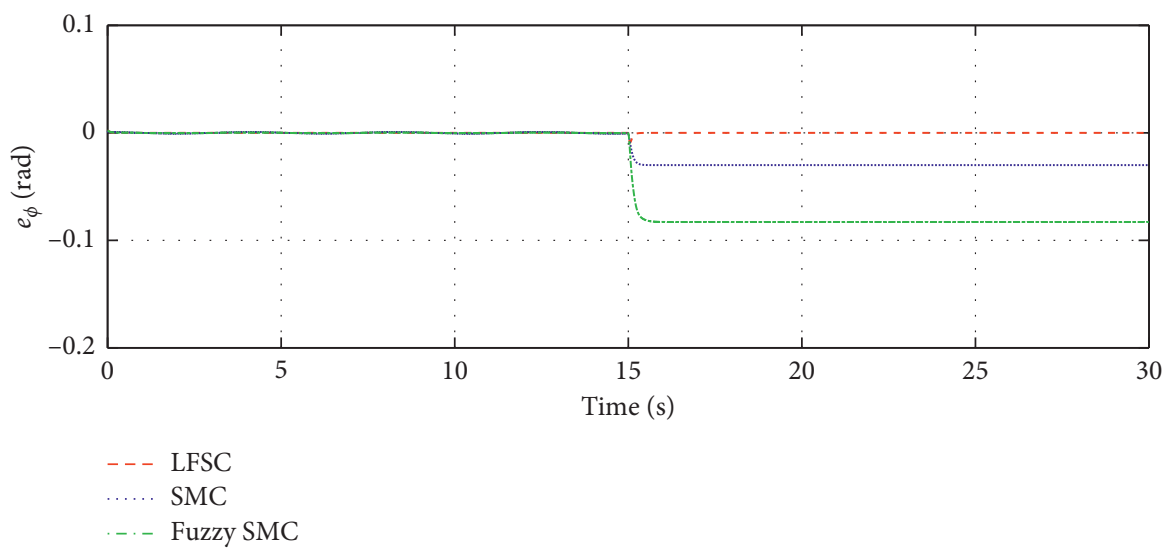

(a)

Figure 14: Continued. 

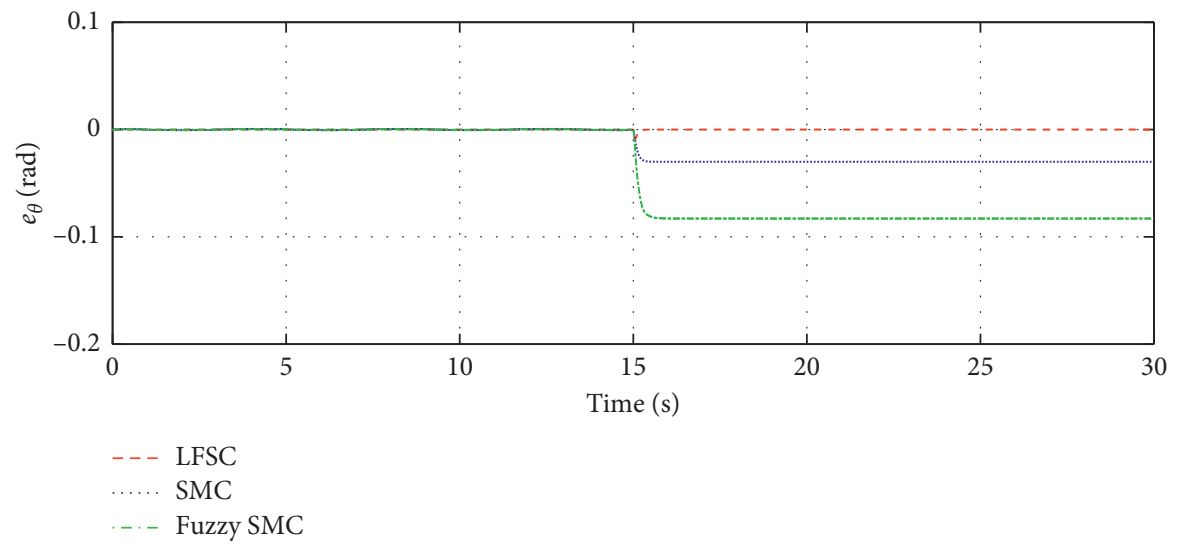

...- Fuzzy SMC

(b)

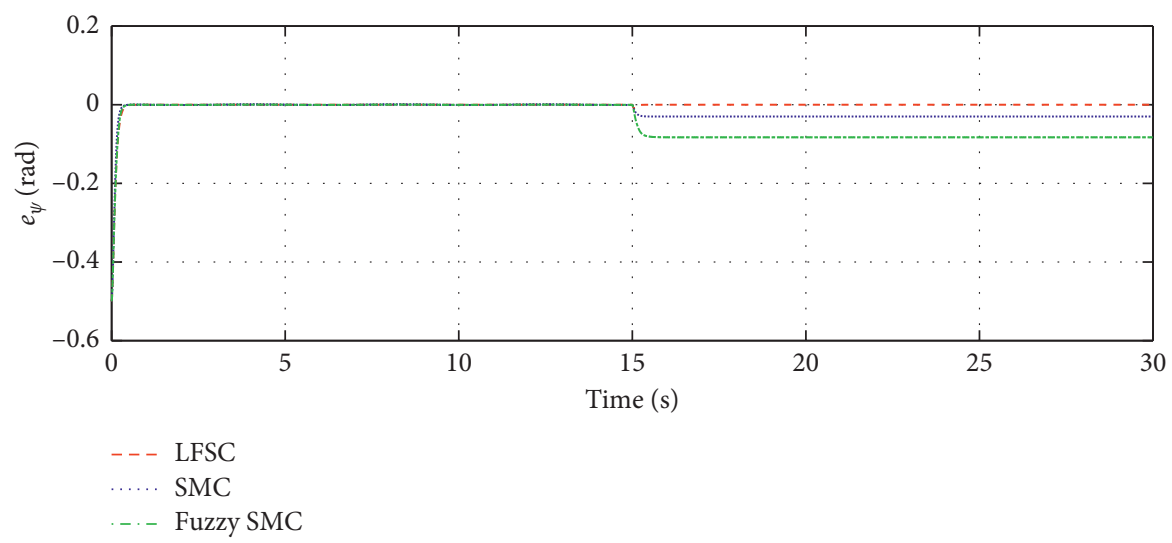

(c)

FIGURE 14: Trajectory tracking errors $\left(e_{\phi}, e_{\theta}, e_{\psi}\right)$.

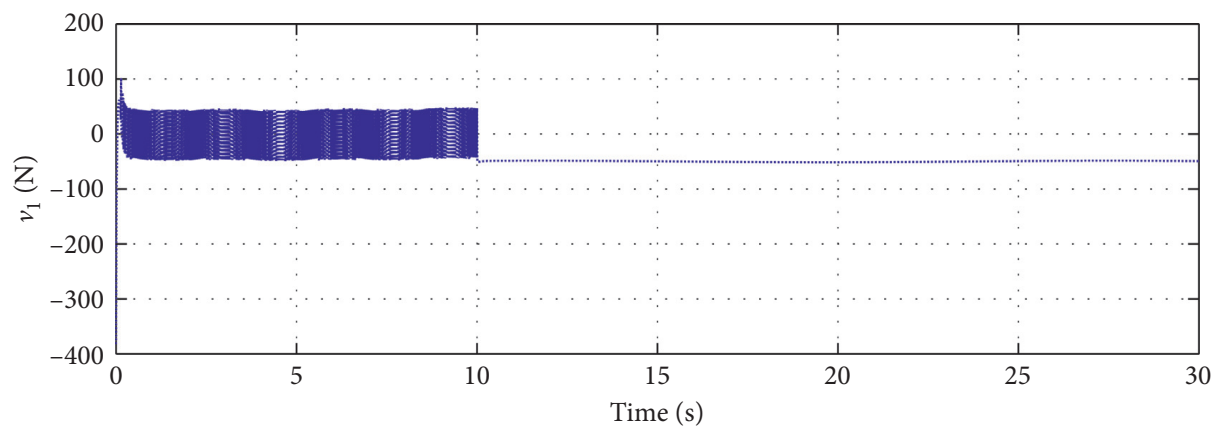

(a)

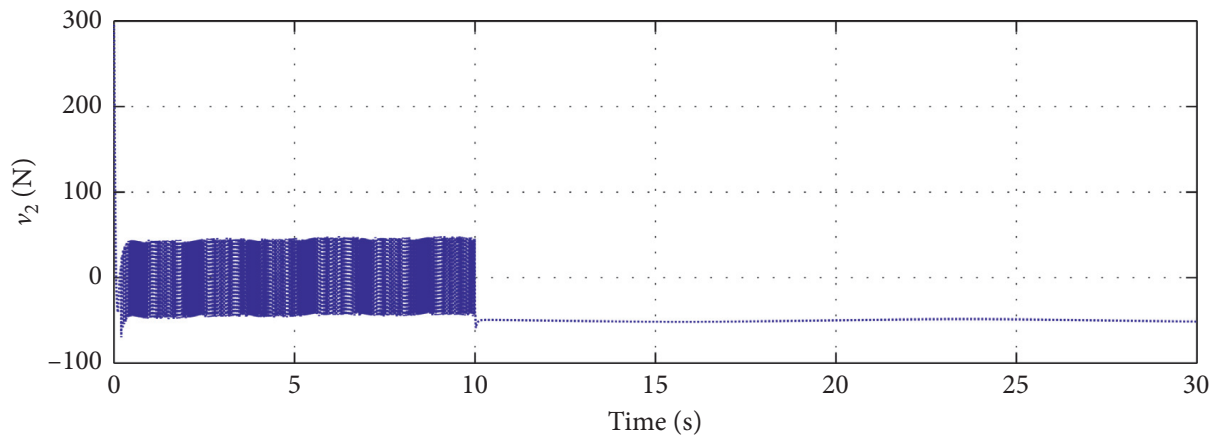

(b)

Figure 15: Continued. 


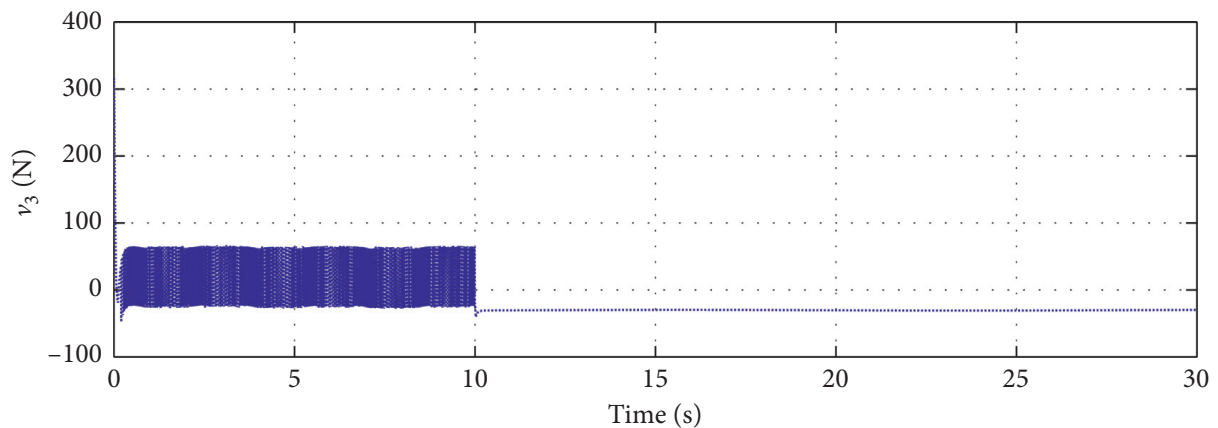

(c)

FIgURE 15: Virtual control input $v_{1}, v_{2}$, and $v_{3}$ of SMC.

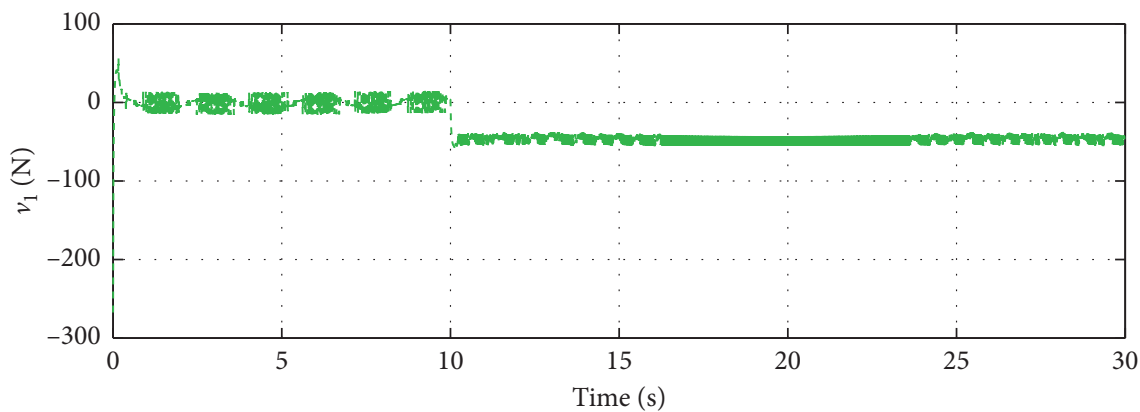

(a)

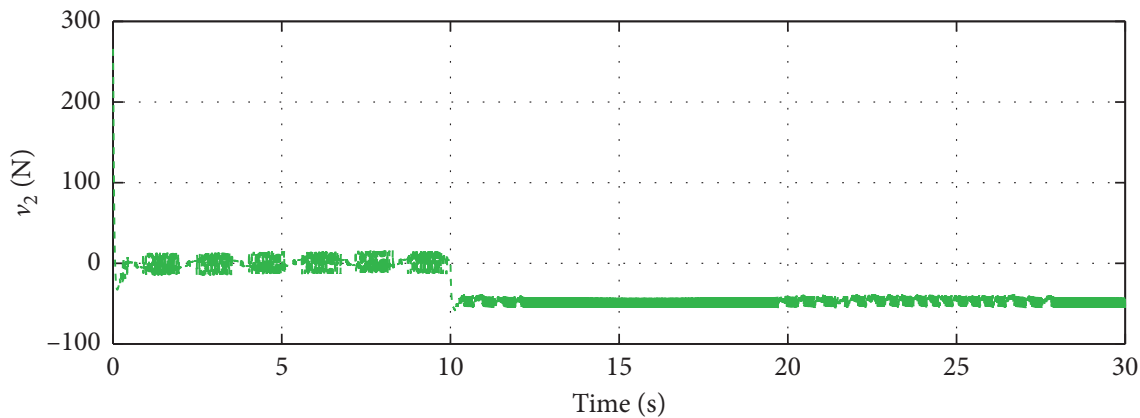

(b)

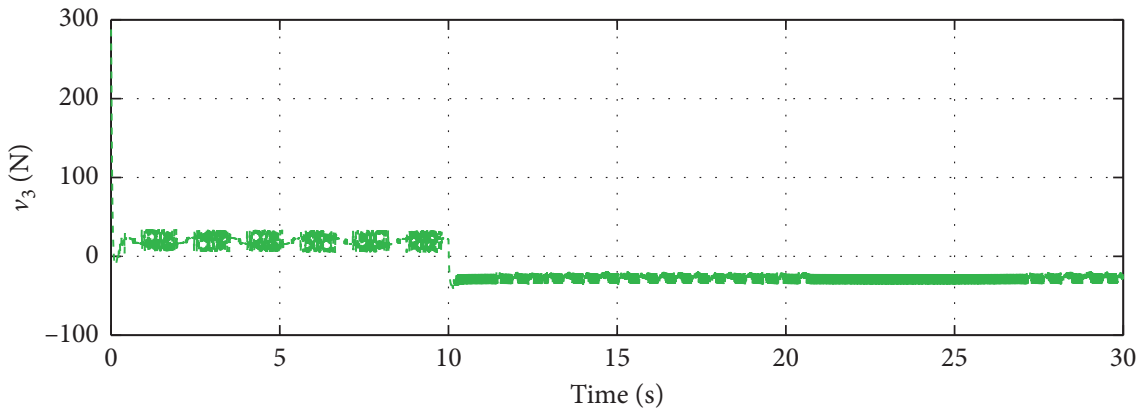

(c)

FIGURE 16: Virtual control input $v_{1}, v_{2}$, and $v_{3}$ of FSMC. 


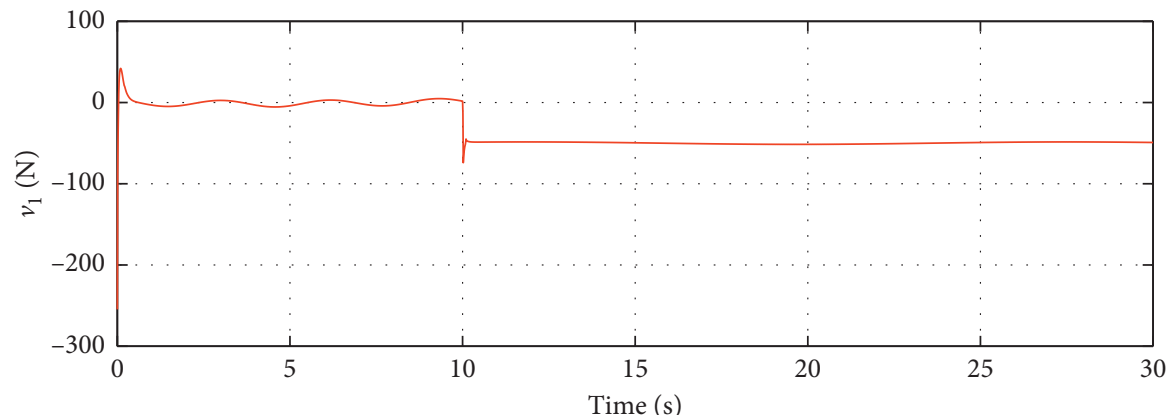

(a)

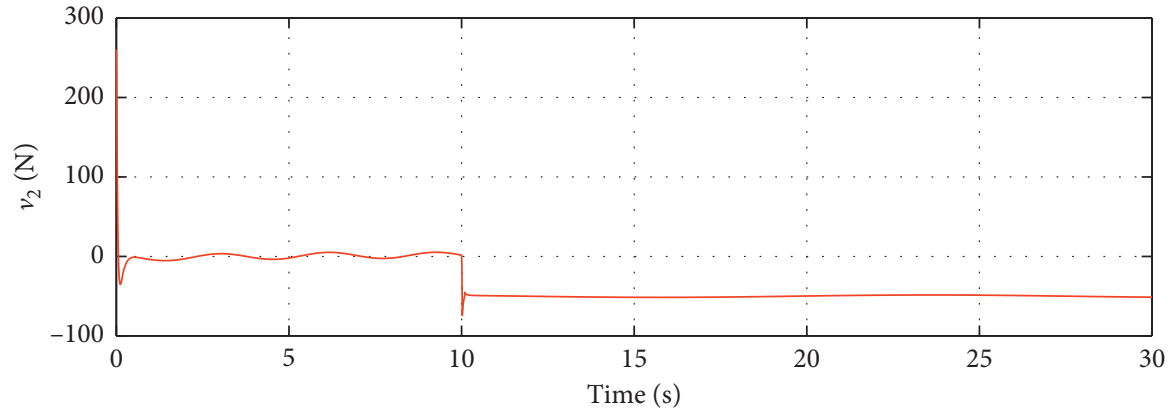

(b)

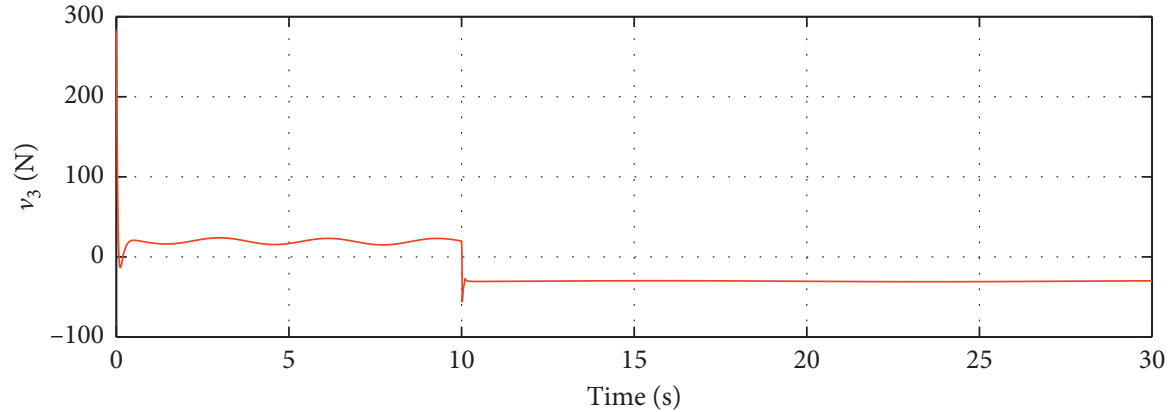

(c)

FIgURE 17: Virtual control input $v_{1}, v_{2}$, and $v_{3}$ of LFSC. 


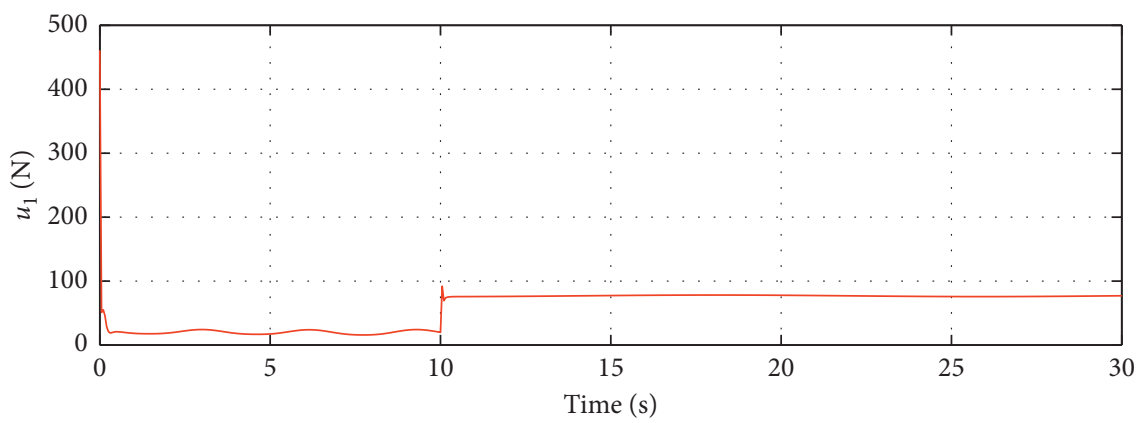

(a)

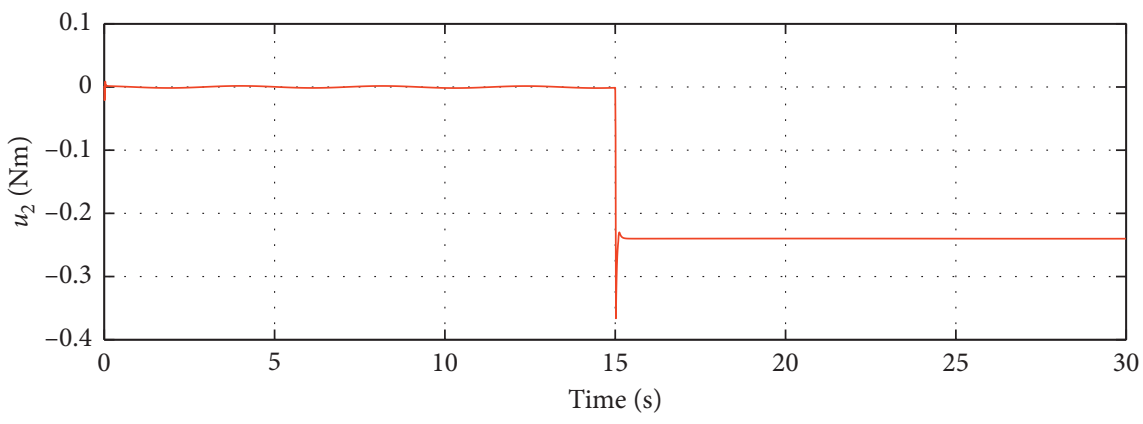

(b)

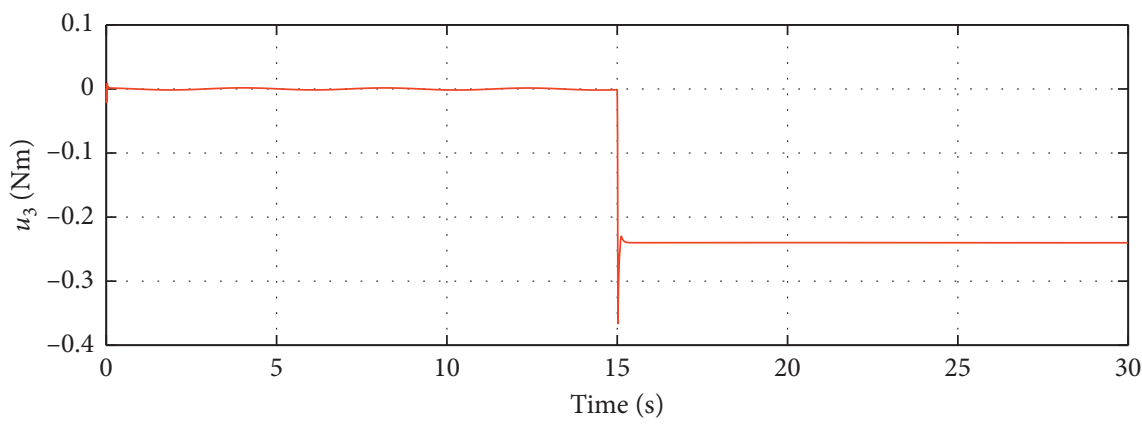

(c)

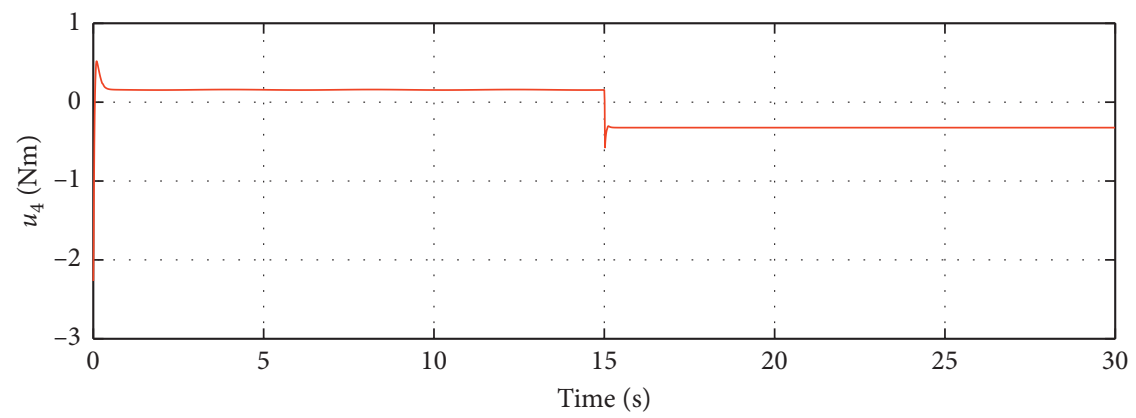

(d)

FIGURE 18: Control inputs $u_{1}, u_{2}, u_{3}$, and $u_{4}$ of LFSC.

\section{Conclusions}

In this paper, an LFSC scheme was presented to guarantee that the tracking errors of all system state variables converge to zero in finite time and eliminate the chattering phenomenon caused by the switching control action, in the presence of external disturbances and uncertainties. Firstly, a linear prediction method was devised to minimize the effects of external disturbances. Secondly, a new fuzzy law was implemented to eliminate the chattering phenomenon. In addition, the stabilities of position and attitude were demonstrated by Lyapunov theory, respectively. Finally, several quadrotor trajectory tracking simulation examples were presented. The control performances obtained using 
traditional SMC and fuzzy SMC schemes were compared to demonstrate the superior performance of the proposed LFSC scheme.

The main conclusions are summarized as follows.

(1) The proposed LFSC scheme can guarantee that the tracking errors of all system state variables converge to zero in finite time.

(2) The high-frequency chattering phenomenon caused by the switching control action does not appear using the proposed LFSC scheme.

(3) Simulation results demonstrate the superior performance and robustness of the proposed LFSC scheme in the case of external disturbances.

(4) Compared with [42, 43], simulations demonstrate the accuracy and superiority of the proposed LFSC method.

The simplicity of the approach, and the use of continuous control signals, makes it readily applicable to a real quadrotor. Advantages of the proposed LFSC method are accuracy, robustness, state variables converge to zero in finite time, and no chattering phenomenon. Therefore, the quadrotor with the LFSC method can be applied to the emergency mission, disaster relief mission, and special military mission. Further work will focus on utilizing the deep learning method to compensate the uncertainties of the system without requirement of their upper bounds and utilizing software ANSYS to research disturbances caused by complex external environments.

\section{Data Availability}

The data used to support the findings of this study are available from the corresponding author upon request.

\section{Conflicts of Interest}

The authors declare that there are no conflicts of interest regarding the publication of this paper.

\section{Acknowledgments}

This work was supported by National Key R\&D Program of China (Grant no. 2017YFC0602000) and Science and Technology Development Project of Jilin Province (Grant no. 20190303061SF).

\section{References}

[1] L. Qian and H. H. T. Liu, "Path-following control of a quadrotor UAV with a cable-suspended payload under wind disturbances," IEEE Transactions on Industrial Electronics, vol. 67, no. 3, pp. 2021-2029, 2020.

[2] J. Sanwale, P. Trivedi, M. Kothari, and A. Malagaudanavar, "Quaternion-based position control of a quadrotor unmanned aerial vehicle using robust nonlinear third-order sliding mode control with disturbance cancellation," in Proceedings of the Institution of Mechanical Engineers Part G-Journal of Aerospace Engineering, vol. 234, pp. 997-1013, Athens, Greece, January 2020.
[3] J. Dong and B. He, "Novel fuzzy PID-type iterative learning control for quadrotor UAV," Sensors, vol. 19, no. 1, 2019.

[4] R. Amin, L. Aijun, and S. Shamshirband, "A review of quadrotor UAV: control methodologies and performance evaluation," International Journal of Automation and Control, vol. 10, no. 2, pp. 87-103, 2016.

[5] F. Chen, R. Jiang, K. Zhang, B. Jiang, and G. Tao, "Robust backstepping sliding-mode control and observer-based fault estimation for a quadrotor UAV," IEEE Transactions on Industrial Electronics, vol. 63, pp. 5044-5056, 2016.

[6] F. Kendoul, "Survey of advances in guidance, navigation, and control of unmanned rotorcraft systems," Journal of Field Robotics, vol. 29, no. 2, pp. 315-378, 2012.

[7] Y. Q. Fan, J. P. Shao, G. T. Sun, and X. Shao, "Improved beetle antennae search algorithm-based levy flight for tuning of PID controller in force control system," Mathematical Problems in Engineering, vol. 2020, Article ID 4287315, , 2020.

[8] N. X. Liu, S. Q. Cao, and J. T. Fei, "Fractional-order PID controller for active power filter using active disturbance rejection control," Mathematical Problems in Engineering, vol. 2019, Article ID 6907570, , 2019.

[9] S. Khatoon, I. Nasiruddin, and M. Shahid, "Design and simulation of a hybrid PD-ANFIS controller for attitude tracking control of a quadrotor UAV," Arabian Journal for Science and Engineering, vol. 42, no. 12, pp. 5211-5229, 2017.

[10] Y. Oda and M. Kumon, "Autonomous flight control of quadrotor helicopter by simple adaptive control with inner loop PD controller," Journal of Robotics and Mechatronics, vol. 30, no. 3, pp. 380-389, 2018.

[11] F. Rinaldi, S. Chiesa, and F. Quagliotti, "Linear quadratic control for quadrotors UAVs dynamics and formation flight," Journal of Intelligent \& Robotic Systems, vol. 70, no. 1-4, pp. 203-220, 2013.

[12] P. Castillo, R. Lozano, and A. Dzul, "Stabilization of a mini rotorcraft with four rotors," IEEE Control Systems, vol. 25, no. 6, pp. 45-55, 2005.

[13] J.-J. Xiong and G.-B. Zhang, "Global fast dynamic terminal sliding mode control for a quadrotor UAV," Isa Transactions, vol. 66, pp. 233-240, 2017.

[14] H. Ma and Y. Li, "A novel dead zone reaching law of discretetime sliding mode control with disturbance compensation," IEEE Transactions on Industrial Electronics, vol. 67, no. 6, pp. 4815-4825, 2020.

[15] K. Wang, C. Hua, J. Chen, and M. Cai, "Dual-loop integral sliding mode control for robust trajectory tracking of a quadrotor," International Journal of Systems Science, vol. 51, no. 2, pp. 203-216, 2020.

[16] M. Morawiec, K. Blecharz, and A. Lewicki, "Sensorless rotor position estimation of doubly fed induction generator based on backstepping technique," IEEE Transactions on Industrial Electronics, vol. 67, no. 7, pp. 5889-5899, 2020.

[17] M. Morawiec and A. Lewicki, "Application of sliding switching functions in backstepping based speed observer of induction machine," IEEE Transactions on Industrial Electronics, vol. 67, no. 7, pp. 5843-5853, 2020.

[18] H. Qi, Y. Shi, S. Li, Y. Tian, D.-L. Yu, and J. B. Gomm, "Fault tolerant control for nonlinear systems using sliding mode and adaptive neural network estimator," Soft Computing, vol. 24, no. 15, pp. 11535-11544, 2020.

[19] K. Liu, H. Gao, H. Ji, and Z. Hao, "Adaptive sliding mode based disturbance attenuation tracking control for wheeled mobile robots," International Journal of Control, Automation and Systems, vol. 18, no. 5, pp. 1288-1298, 2020. 
[20] C. Zhang, G. Zhang, and Q. Dong, "Multi-variable finite-time observer-based adaptive-gain sliding mode control for fixedwing UAV," IET Control Theory \& Applications, vol. 15, no. 2, pp. 223-247, 2021.

[21] X. Lin, Y. Yu, and C.-y. Sun, "A decoupling control for quadrotor UAV using dynamic surface control and sliding mode disturbance observer," Nonlinear Dynamics, vol. 97, no. 1, pp. 781-795, 2019.

[22] S. Mobayen, "Finite-time robust-tracking and model-following controller for uncertain dynamical systems," Journal of Vibration and Control, vol. 22, no. 4, pp. 1117-1127, 2016.

[23] T. Yoshimura, "Discrete-time adaptive sliding mode control for uncertain systems based on multi-rate output measurement," International Journal of Systems Science, vol. 44, no. 9, pp. 1733-1744, 2013.

[24] J. Zhang, H. Wang, M. Ma, M. Yu, A. Yazdani, and L. Chen, "Active front steering-based electronic stability control for steer-by-wire vehicles via terminal sliding mode and extreme learning machine," IEEE Transactions on Vehicular Technology, vol. 69, no. 12, pp. 14713-14726, 2020.

[25] M. Zhou, Y. Feng, C. Xue, and F. Han, "Deep convolutional neural network based fractional-order terminal sliding-mode control for robotic manipulators," Neurocomputing, vol. 416, pp. 143-151, 2020.

[26] Y. Song, H. Li, and X. Shi, "Stabilization of a class of nonlinear underactuated robotic systems through nonsingular fast terminal sliding mode control," Mathematical Problems in Engineering, vol. 2020, Article ID 5426087, , 2020.

[27] X. Gu, Y. Niu, and B. Chen, "Adaptive non-singular fast terminal sliding mode control for multi-agent systems with unknown non-linear dynamics," IET Control Theory \& Applications, vol. 14, no. 16, pp. 2223-2232, 2020.

[28] G. Chen, B. Jin, and Y. Chen, "Accurate and robust body position trajectory tracking of six-legged walking robots with nonsingular terminal sliding mode control method," Applied Mathematical Modelling, vol. 77, pp. 1348-1372, 2020.

[29] J.-J. Xiong, G.-B. Zhang, J.-X. Wang, and T.-H. Yan, "Improved sliding mode control for finite-time synchronization of nonidentical delayed recurrent neural networks," IEEE Transactions on Neural Networks and Learning Systems, vol. 31, no. 6, pp. 2209-2216, 2020.

[30] J. A. González, A. Barreiro, S. Dormido, and A. Baños, "Nonlinear adaptive sliding mode control with fast nonovershooting responses and chattering avoidance," Journal of the Franklin Institute, vol. 354, no. 7, pp. 2788-2815, 2017.

[31] N. Wang, Q. Deng, G. Xie, and X. Pan, "Hybrid finite-time trajectory tracking control of a quadrotor," Isa Transactions, vol. 90, pp. 278-286, 2019.

[32] S. Mallavalli and A. Fekih, "An SMC-based fault tolerant control design for a class of underactuated unmanned aerial vehicles," in Proceedings of the 2018 4th International Conference on Control, Automation and Robotics (ICCAR), pp. 152-155, IEEE, Auckland, New Zealand, April 2018.

[33] H. Wang, X. Ye, Y. Tian, G. Zheng, and N. Christov, "Modelfree-based terminal SMC of quadrotor attitude and position," IEEE Transactions on Aerospace and Electronic Systems, vol. 52, no. 5, pp. 2519-2528, 2016.

[34] X. H. Xinghuo Yu and Z. H. Man Zhihong, "Fast terminal sliding-mode control design for nonlinear dynamical systems," IEEE Transactions on Circuits and Systems I: Fundamental Theory and Applications, vol. 49, no. 2, pp. 261-264, 2002.
[35] Z. Li, X. Ma, and Y. Li, "Robust tracking control strategy for a quadrotor using RPD-SMC and RISE," Neurocomputing, vol. 331, pp. 312-322, 2019.

[36] F. Yacef, O. Bouhali, M. Hamerlain, and N. Rizoug, "Observer-based adaptive fuzzy backstepping tracking control of quadrotor unmanned aerial vehicle powered by li-ion battery," Journal of Intelligent \& Robotic Systems, vol. 84, no. 1-4, pp. 179-197, 2016.

[37] Y. Naidoo, R. Stopforth, and G. Bright, "Quad-rotor unmanned aerial vehicle helicopter modelling \& control," International Journal of Advanced Robotic Systems, vol. 8, pp. 139-149, 2011.

[38] S. Bouabdallah and R. Siegwart, "Full control of a quadrotor," in Proceedings of the 2007 IEEE/RSJ International Conference on Intelligent Robots and Systems, pp. 153-158, IEEE, San Diego, CA, USA, October 2007.

[39] A. L. Salih, M. Moghavvemi, H. A. F. Mohamed, and K. S. Gaeid, "Modelling and PID controller design for a quadrotor unmanned air vehicle," in Proceedings of 2010 IEEE International Conference on Automation, Quality and Testing, Robotics, Cluj-Napoca, Romania, May 2010.

[40] E.-H. Zheng, J.-J. Xiong, and J.-L. Luo, "Second order sliding mode control for a quadrotor UAV," Isa Transactions, vol. 53, no. 4, pp. 1350-1356, 2014.

[41] J. Lv, J. Shen, H. Xia et al., "Fuzzy SMC for UAV to resist strong wind with disturbance approximation," in Proceedings of the 2018 Ninth International Conference on Intelligent Control and Information Processing (ICICIP), pp. 93-98, IEEE, Wanzhou, China, November 2018.

[42] G. Xu, Y. Xia, D. H. Zhai, and D. Ma, “Adaptive prescribed performance terminal sliding mode attitude control for quadrotor under input saturation," IET Control Theory \& Applications, vol. 14, no. 17, pp. 2473-2480, 2020.

[43] G. B. Wang, "Adaptive sliding mode robust control based on multi-dimensional Taylor network for trajectory tracking of quadrotor UAV," IET Control Theory \& Applications, vol. 14, no. 14, pp. 1855-1866, 2020. 